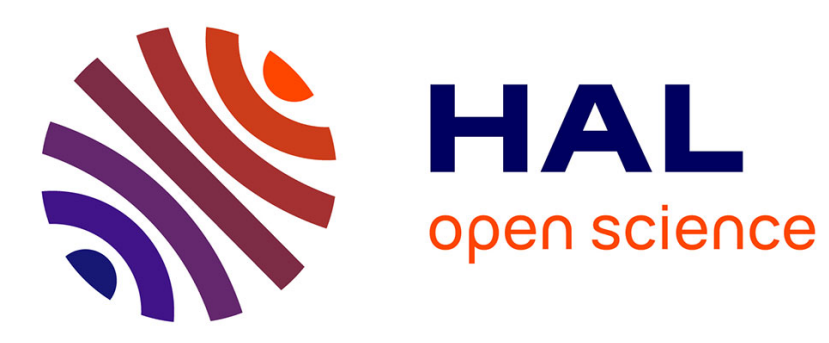

\title{
Stability of the columnar and smectic phases of length-bidisperse parallel hard cylinders
}

\author{
Szabolcs Varga, Enrique Velasco, Luis Mederos, Franz J Vesely
}

\section{To cite this version:}

Szabolcs Varga, Enrique Velasco, Luis Mederos, Franz J Vesely. Stability of the columnar and smectic phases of length-bidisperse parallel hard cylinders. Molecular Physics, 2009, 107 (23-24), pp.2481-2492. 10.1080/00268970903369180 . hal-00548165

\section{HAL Id: hal-00548165 \\ https://hal.science/hal-00548165}

Submitted on 19 Dec 2010

HAL is a multi-disciplinary open access archive for the deposit and dissemination of scientific research documents, whether they are published or not. The documents may come from teaching and research institutions in France or abroad, or from public or private research centers.
L'archive ouverte pluridisciplinaire HAL, est destinée au dépôt et à la diffusion de documents scientifiques de niveau recherche, publiés ou non, émanant des établissements d'enseignement et de recherche français ou étrangers, des laboratoires publics ou privés. 


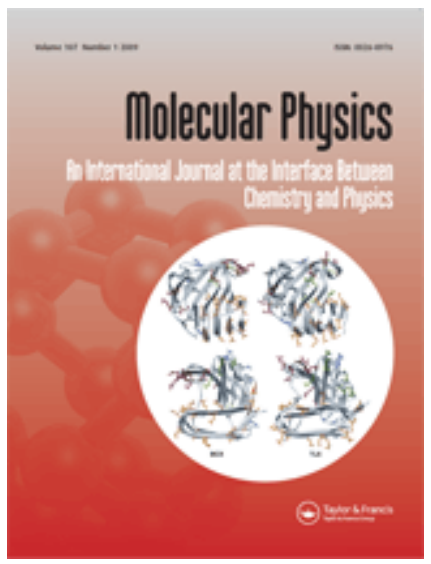

\section{Stability of the columnar and smectic phases of length- bidisperse parallel hard cylinders}

\begin{tabular}{|r|l|}
\hline Journal: & Molecular Physics \\
\hline Manuscript ID: & TMPH-2009-0217.R1 \\
\hline Manuscript Type: & Full Paper \\
\hline Date Submitted by the \\
Author: & $01-$ Sep-2009 \\
\hline Complete List of Authors: & $\begin{array}{l}\text { Varga, Szabolcs; University of Pannonia, Institute of Physics } \\
\text { Velasco, Enrique; UAM, Departamento de Física Teórica de la } \\
\text { Materia Condensada } \\
\text { Mederos, Luis; Instituto de Ciencia de Materiales de Madrid } \\
\text { Vesely, Franz; University of Vienna }\end{array}$ \\
\hline Keywords: & $\begin{array}{l}\text { liquid crystal, binary mixture, phase behaviour, Onsager theory, } \\
\text { nematic,smectic, columnar }\end{array}$ \\
\hline \multicolumn{2}{|c}{} \\
\hline
\end{tabular}

\section{scholarONE" \\ Manuscript Central}




\title{
Stability of the columnar and smectic phases of length-bidisperse
}

\author{
parallel hard cylinders
}

\author{
Szabolcs Varga ${ }^{a)}$, Enrique Velasco ${ }^{b)}$, Luis Mederos ${ }^{c)}$ and Franz Vesely ${ }^{d)}$ \\ ${ }^{a)}$ Institute of Physics, University of Pannonia, PO Box 158, Veszprém, H-8201 Hungary \\ ${ }^{b)}$ Departamento de Física Teórica de la Materia Condensada and Instituto de Ciencia de \\ Materiales Nicolás Cabrera, Universidad Autónoma de Madrid, E-28049 Madrid, Spain \\ c) Instituto de Ciencia de Materiales de Madrid, Consejo Superior de Investigaciones \\ Científicas, Sor Juana Inés de la Cruz, 3, E-28049 Madrid, Spain

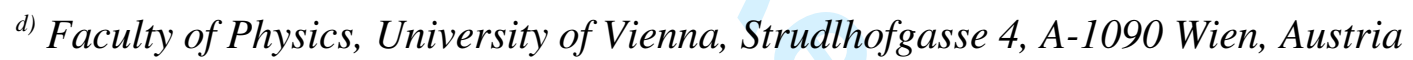

Pacs numbers: 64.70.Md, 64.60.Cn, 61.30.Cz, 61.30.St

Number of pages: 40 (including figures)

Figures: 7 
Abstract

The effect of length-bidispersity on the stability of nematic, smectic and columnar phases of rod-like particles is studied in the perfect alignment limit using the Onsager's second virial theory. The rod-like particles are modeled as hard cylinders of equal diameters (D) but different lengths $\left(L_{1} \neq L_{2}\right)$. Three different smectic structures are observed: (i) the conventional smectic $\left(S_{1}\right)$ phase, where both components accommodate in the same layer; (ii) the microsegregated smectic $\left(\mathrm{S}_{2}\right)$ phase, which can be considered as an alternation of fluid layers rich in short and long rods, respectively; and (iii) two layers of short rods accommodate inside one layer of long rods, which gives the third smectic $\left(S_{3}\right)$ structure. Due to the inefficient packing of the short and long rods into a layered structure along the symmetry axes of the rods, the smectic phase is destabilized with respect to nematic and columnar phases upon mixing the short and long components. With decreasing length ratio $\left(l=L_{2} / L_{1}\right)$ the smectic phase is destabilized with respect to the nematic phase at compositions rich in short rods and two forms of smectic phases, namely $S_{1}$ and $S_{2}$, take place in alternation. The alternation of the structure is the consequence of the minimization of the number of overlapping layers of the short rods with one long rod. In mixtures rich in long rods, the short and long rods are in the same layer up to $l=0.39$, while the short rods can accommodate into the interstitial region of long rods for $l<0.39$ and the system forms a $S_{2}$ phase. The $S_{3}$ phase is observed in the range $0.57<l<0.39$ and is due to the efficient packing of two layers of short rods inside one smectic layer of long rods. Our theoretical predictions for the three smectic structures are in close agreement with the smectic phase behaviour of a binary mixture of short and long helical polysilanes (Okashi et al., Macromolecules 42, 3443 (2009)). It seems reasonable that the helical polysilanes can be considered as an ideal system for testing the hard-body theories. Finally, it is interesting that the stabilization process of the columnar phase with respect to the smectic ordering with decreasing length ratio turns over at $l=0.3$. 


\section{Introduction}

Liquids and colloidal suspensions consisting of linear particles display an extremely interesting phase behaviour that becomes even more pronounced as we proceed from monodisperse systems to mixtures. In addition to the nematic, smectic and solid structures encountered in the pure substances, fractionation and demixing transitions [1-7], microsegregated smectic as well as columnar phases may occur in mixtures [8-18]. Frenkel [19] was the first to point out that an entropy-driven columnar phase transition is possible even in monodisperse systems, although only for disc-shaped particles such as cut spheres, while elongated particles such as spherocylinders and cylinders display only isotropic, nematic and smectic (and crystalline) phases [20-22]. Further insight into the stability of the nematic, smectic and solid phases in systems of identical parallel cylinders was provided by Mori and Kimura [23] and Xu et al. [24]. Microsegregation of different rods in the smectic phase was first reported by Koda and Kimura [12] who applied the second virial approximation to parallel cylinders. Following this line of research, Koda et al. investigated mixtures of parallel rods and spheres, both by Monte Carlo simulation and by basic density functional theory [14-15]. Further studies of rod-sphere systems followed by Dogic et al. [16], Cinacchi et al. [6] and Vesely [18]. The first account of a suppression of the smectic phase is due to Sluckin, who studied a length-polydisperse mixture of hard rods [8]. Evidence of the importance of the columnar phase was then explored by several simulation and theoretical studies [9-11,13,25-28]. In a sequence of extensive simulation studies, Stroobants $[9,10]$ demonstrated the presence of a columnar phase in mixtures of long and short rods. Sear and Jackson [13] applied second virial theory and bifurcation analysis to parallel bidisperse cylinders, demonstrating the possibility of a nematic-columnar transition masking the smectic phase. Both MC simulation and theoretical methods were used by van Roij et al. $[3,26]$ to 
investigate the demixing of short and long rods in the isotropic and nematic phases, as well as the smectic and columnar bifurcations. Cui and Chen [11] and Bohle et al. [25] applied bifurcation analysis and Landau theory to study the ordered phases of bi- and polydisperse rods, while Bates and Frenkel used semigrand MC simulation, again demonstrating the presence - and possible dominance - of a columnar phase [27]. In the recent FMT study of Martínez-Ratón and Cuesta [28] the dominance of the columnar phase is confirmed above a threshold value of the polydispersity in a system of parallel cylinders.

Several rod-like colloidal dispersions have been prepared to examine by experiment the effect of hard body interactions in the stabilization of the liquid crystalline mesophases. Well-known examples are polydisperse mineral liquid crystals [29-30], the mixture of colloidal spheres and TMV viruses [31], the binary mixture of thin and thick fd viruses [5] and solutions of single walled carbon nanotubes [32-34]. A common feature of the mineral rods and the carbon nanotubes is that they are polydisperse mainly with respect to length. To give a theoretical explanation for the phase behaviour of polydisperse rods, it is customary to use continuous distribution functions such the Shultz-Flory distribution in Onsager and related theories [35-36]. Instead of using continuous distribution functions one may consider the system as a binary mixture of short and long rods, which leads to qualitatively correct results for the phase transitions. For instance, the isotropic-nematic-nematic three phase coexistence of a dispersion of sterically stabilized rodlike boehmite particles can be explained by Onsager theory of short and long rods [30].

In a foregoing study we have investigated mixtures of hard elongated particles with equal length but differing diameters [37]. As a natural continuation of our previous work, the present work is devoted to mixtures of parallel cylinders having equal diameters but different lengths. The experimental motivation of our work is the very recent study of the smectic phase in binary mixtures of short and long helical polysilanes [38]. The main feature of the 
polysilanes is that excluded volume interactions are dominant, electrostatic and the van der Waals interactions are weak, and almost monodisperse solutions can be prepared. Due to these advantageous properties the polysilanes can be considered as an ideal playground for testing the Onsager and other density-functional theories. Okoshi et al. [38] have focused on determining the possible smectic structures of binary mixtures of polysilanes using x-ray scattering and atomic force microscopy. In addition to the conventional and the microsegregated smectic phases, a new finely structured smectic phase was discovered. We make an attempt to find this new smectic structure in our present study. Another reason of our work is that the relative stability of the columnar, smectic and nematic phases in various regions of parameter space has not yet been studied thoroughly in MC simulation and theoretical studies. It is clear that the columnar phase is an important though somewhat elusive feature in the interplay of ordered phases in mixtures of elongated particles. A nematic-columnar transition may preempt the formation of a smectic phase, and microsegregation may be delayed by the formation of well-mixed columnar structures. At high densities, even the transition to a solid is sometimes preceded by the formation of a columnar phase of larger entropy. It is the purpose of this study to investigate the relative stabilities of the ordered and demixed states of bidisperse parallel cylinder systems, using a method that provides higher accuracy and reliability than the bare bifurcation approaches. 


\section{Model system and Onsager theory for smectic and columnar phases}

We consider the binary mixture of parallel hard cylinders, where both components have equal diameters $\left(D=D_{1}=D_{2}\right)$ while their lengths are different $\left(L_{1} \neq L_{2}\right)$. Restricting ourselves only to nematic, smectic and columnar phases, it is useful to decompose the positional vector of the cylinders $(\vec{r})$ as a sum of mutually perpendicular vectors $\vec{r}_{\perp}$ and $\vec{r}_{\|}$. We use $\vec{r}_{\perp}=(x, y, 0)$ for the description of the columnar phase, while $\vec{r}_{\|}=(0,0, z)$ is for the smectic one, the long axes of the cylinders being orientated along the z-axis. In this way the columnar phase is a two-dimensional solid in the $\mathrm{x}-\mathrm{y}$ plane, while the smectic is a onedimensional solid along the $z$ axis. The corresponding local columnar and smectic densities for binary mixtures are $\rho_{i}\left(\vec{r}_{\perp}\right)$ and $\rho_{i}\left(\vec{r}_{\|}\right)(i=1,2)$, respectively. The ideal Helmholtz free energy density of $N=N_{1}+N_{2}$ particles in a vessel of volume $V$ for the binary mixture can be written for the columnar phase as

$$
\frac{\beta F_{i d}}{V}=\frac{1}{A} \sum_{i=1}^{2} \int_{A} d \vec{r}_{\perp} \rho_{i}\left(\vec{r}_{\perp}\right)\left\{\ln \left(\Lambda_{i}^{3} \rho_{i}\left(\vec{r}_{\perp}\right)\right)-1\right\},
$$

where $\beta=\frac{1}{k_{B} T}$ (T being the temperature and $k_{B}$ the Boltzmann constant), $\rho_{i}=N_{i} / V$ is the local number density of component $i \quad(i=1,2), \Lambda_{i}^{3}$ is the de Broglie volume of each component, including the translational and rotational contributions to the kinetic energy, and $A=\frac{\sqrt{3}}{2} a^{2}$ is the area of the hexagonal unit cell. In the smectic phase the ideal contribution of the free energy is simpler and can be written as a one-dimensional integral as follows

$$
\frac{\beta F_{i d}}{V}=\frac{1}{a} \sum_{i=1}^{2} \int_{0}^{a} d z \rho_{i}(z)\left\{\ln \left(\Lambda_{i}^{3} \rho_{i}(z)\right)-1\right\},
$$

where $a$ is now the smectic period. 
The residual contribution of the Helmholtz free energy is approximated by the second viral contribution in the virial series $[39,40]$

$$
\beta F_{r e s}=-\frac{1}{2} \sum_{i, j=1}^{2} \int d \vec{r}_{1} \rho_{i}\left(\vec{r}_{1}\right) \int d \vec{r}_{2} \rho_{j}\left(\vec{r}_{2}\right) f_{M}^{i j}\left(\vec{r}_{12}\right),
$$

where the Mayer function $\left(f_{M}^{i j}\right)$ of the hard cylinders can be written as a product of Heaviside functions of columnar and smectic variables,

$$
f_{M}^{i j}(\vec{r})=-\theta\left(D-r_{\perp}\right) \theta\left(L_{i j}-|z|\right)
$$

with $r_{\perp}=\left|\vec{r}_{\perp}\right|$ and $L_{i j}=\frac{L_{i}+L_{j}}{2}$. Substituting Eq. (3) into Eq. (2) only the $z$ spatial variable can be integrated out for the columnar phase, and $x$ and $y$ for the smectic order. The resulting residual free energy densities for the columnar and the smectic phases are

$$
\begin{aligned}
& \frac{\beta F_{r e s}}{V}=\frac{1}{A} \sum_{i, j=1}^{2} L_{i j} \int_{A} d \vec{r}_{\perp 1} \rho_{i}\left(\vec{r}_{\perp 1}\right) \int d \vec{r}_{\perp 2} \rho_{j}\left(\vec{r}_{\perp 2}\right) \theta\left(D-r_{\perp 12}\right), \\
& \frac{\beta F_{r e s}}{V}=\frac{1}{2 a} \sum_{i, j=1}^{2} D^{2} \pi \int_{0}^{a} d z_{1} \rho_{i}\left(z_{1}\right) \int d z_{2} \rho_{j}\left(z_{2}\right) \theta\left(L_{i j}-\left|z_{12}\right|\right) .
\end{aligned}
$$

The sum of the corresponding equations of Eq. (1) and Eq. (4) constitutes our total Helmholtz free energy density. The equilibrium structure of the binary mixture is determined by that density profile which minimizes the free energy of the system. In this work we use the method of Fourier parametrization for both columnar and smectic ordering. Instead of the local number densities, we parametrize the positional distribution functions given by the ratio of local and mean number densities, i.e. $f_{i}(\vec{r})=\rho_{i}(\vec{r}) / \rho_{i}(i=1,2)$.

In the columnar phase the Fourier representation of $f_{i}\left(\vec{r}_{\perp}\right)$ is given by

$$
f_{i}\left(\vec{r}_{\perp}\right)=\sum_{n_{1} n_{2}} f_{i, n_{1} n_{2}} \cos \left(n_{1} \vec{q}_{1} \cdot \vec{r}_{\perp}+n_{2} \vec{q}_{2} \cdot \vec{r}_{\perp}\right)
$$


where $f_{i, n_{1} n_{2}}$ is the Fourier coefficient of component $i, n_{1}$ and $n_{2}$ are integer numbers, and $\vec{q}_{1}, \vec{q}_{2}$ are reciprocal lattice vectors. The reciprocal lattice vectors of the hexagonal phase can be obtained by the orthogonality relation between the unit and reciprocal vectors. These vectors are $\vec{q}_{1}=\left(q,-\frac{q}{\sqrt{3}}, 0\right)$ and $\vec{q}_{2}=\left(0, \frac{2 q}{\sqrt{3}}, 0\right)$, where $q=\frac{2 \pi}{a}$. The simple form of Eq. (5) is due to the inversion symmetry of the columnar phase: $f_{i}\left(\vec{r}_{\perp}\right)=f_{i}\left(-\vec{r}_{\perp}\right)$. Inserting Eq. (5) into Eq. (4a) and using the orthogonality relations of the cosine and sine functions one obtains that

$$
\frac{\beta F_{r e s}}{V}=4 D^{2} \sum_{i, j, n_{1} n_{2}} L_{i j} \rho_{i} \rho_{j} f_{i, n_{1} n_{2}} f_{j, n_{1} n_{2}} \Gamma_{n_{1} n_{2}}(q D)
$$

where $\Gamma_{n_{1} n_{2}}(q D)=\int_{0}^{1} d x j_{0}\left(\left(2 n_{2}-n_{1}\right) \sqrt{\frac{1-x^{2}}{3}} q D\right) \sqrt{1-x^{2}} \cos \left(n_{1} q D x\right)$ and $j_{0}(x)=\sin (x) / x$.

$\Gamma_{n_{1} n_{2}}(q D)$ can be calculated analytically in some special cases, for example $\Gamma_{00}=\frac{\pi}{4}, \Gamma_{2 n, n}=\frac{\pi}{2} \frac{J_{1}(n q D)}{n q D}$. The ideal contribution of the free energy Eq. (1a) cannot be simplified further by the substitution of Eq. (5), but it becomes now a function of the Fourier coefficients. Since the total free energy density, which is the sum of Eqs. (1a) and (6), depends on the Fourier components and the wave number $q$, the equilibrium columnar structure can be determined by the following set of equations

$$
\frac{\partial\left(\frac{\beta F}{V}\right)}{\partial f_{i, n_{1} n_{2}}}=0, \frac{\partial\left(\frac{\beta F}{V}\right)}{\partial q}=0
$$

where $i=1,2$ and $-n \leq n_{1} \leq n,-n \leq n_{2} \leq n$. The normalization constraints of the number densities $\frac{1}{A} \int_{A} d \vec{r}_{\perp} \rho_{i}\left(\vec{r}_{\perp}\right)=\rho_{i}$ determine the values of the zeroth order Fourier coefficients; the results $f_{1,00}=1$ and $f_{2,00}=1$ hold for nematic and columnar phases, too. In the nematic phase 
all Fourier components are zero except $f_{1,00}$ and $f_{2,00}$, while $f_{1, n_{1} n_{2}} \neq 0$ and $f_{2, n_{1} n_{2}} \neq 0$ in the columnar phase. The Fourier coefficients decay rapidly with increasing $n_{1}$ and $n_{2}$. In our calculations it has been more than sufficient to cut the series at the $6^{\text {th }}$ shell, i.e. $n=6$, which guarantees that I $f_{i, n n} \mathrm{k} \varepsilon$, with $\varepsilon=10^{-4}$.

To include the possible in-phase and out-of-phase ordering of the short and long rods in the smectic phase we use the following Fourier ansatz for the long and short rods

$$
\begin{aligned}
& f_{1}(z)=\sum_{i=0}^{m} f_{1, i} \cos (i q z), \\
& f_{2}(z)=\sum_{i=0}^{m} f_{2, i} \cos \left(i q z-i \varphi_{0}\right),
\end{aligned}
$$

where the phase shift $\varphi_{0}$ is zero for in-phase order, while it is $\pi$ for out-of-phase order (microsegregation). We consider the Fourier components $\left(f_{1, i}\right.$ and $\left.f_{2, i}\right)$, wave number $\left(q=\frac{2 \pi}{a}\right)$ and the phase shift $\left(\varphi_{0}\right)$ as free variables of the smectic phase. Using Eqs. (8a-8b) in Eq. (4b) we can derive the residual free energy density in terms of our free variables

$$
\begin{aligned}
& \frac{\beta F_{\text {res }}}{V}=\frac{1}{2} \sum_{i, j=1}^{2} \rho_{i} \rho_{j} V_{e x c}^{i j}+\frac{1}{2} D^{2} \pi \times \\
& \times\left\{\rho_{1}^{2} \sum_{i=1}^{m} f_{1, i}^{2} \frac{\sin \left(i q L_{1}\right)}{i q}+2 \rho_{1} \rho_{2} \sum_{i=1}^{m} f_{1, i} f_{2, i} \frac{\sin \left(i q\left(\frac{L_{1}+L_{2}}{2}\right)\right)}{i q} \cos \left(i \varphi_{0}\right)+\rho_{2}^{2} \sum_{i=1}^{m} f_{2, i}^{2} \frac{\sin \left(i q L_{2}\right)}{i q}\right\},
\end{aligned}
$$

where $V_{e x c}^{i j}$ denotes the excluded volume between a particle of component $i$ and another particle of component $j$. It can be shown easily that $V_{e x c}^{i j}=\frac{\pi}{4}\left(D_{i}+D_{j}\right)^{2}\left(L_{i}+L_{j}\right)$. As for the columnar phase, the ideal contribution of the free energy Eq. (1b) cannot be simplified further by the help of Eq. (8) and we determine it numerically. The free energy of the smectic phase, 
which is the sum of Eq. (1b) and Eq. (9), is minimized with respect to the free variables to obtain the equilibrium structure and the free energy of the smectic phase. The minimization yields the following set of equations

$$
\frac{\partial\left(\frac{\beta F}{V}\right)}{\partial f_{i, j}}=0, \frac{\partial\left(\frac{\beta F}{V}\right)}{\partial q}=0, \frac{\partial\left(\frac{\beta F}{V}\right)}{\partial \varphi_{0}}=0
$$

where $i=1,2$ and $j=1, \ldots, m$. From the normalization condition of the number densities $\frac{1}{a} \int_{0}^{a} d z \rho_{i}(z)=\rho_{i}$ it is trivial to prove that $f_{1,0}=1$ and $f_{2,0}=1$. The value of $m$ is set such that the order of magnitude of $f_{i, m}$ cannot be more than $10^{-4}$. In our calculations the maximum value of $m$ has not exceeded 16 even at very high pressures.

In summary, Eq. (7) determines the free energy of the columnar phase, while Eq. (10) is for the smectic phase. For the case of vanishing Fourier coefficients both the columnar free energy (sum of Eqs. (1a) and (6)) and the smectic free energy [sum of Eqs. (1b) and (9)] reproduces the free energy of the nematic phase.

Having obtained the free energy of the nematic, smectic and columnar phases we can determine the pressure and chemical potentials from the standard thermodynamic equations as follows

$$
\beta \mu_{i}=\frac{\partial\left(\frac{\beta F}{V}\right)}{\partial \rho_{i}}, \beta P=-\frac{\beta F}{V}+\sum_{i=1}^{2} \rho_{i} \frac{\partial\left(\frac{\beta F}{V}\right)}{\partial \rho_{i}} \quad(i=1,2)
$$

These equations are used to determine the phase boundary between two coexisting phases $\alpha_{1}$ and $\alpha_{2}$ from the conditions of the phase coexistence $\beta \mu_{i}^{\alpha_{1}}=\beta \mu_{i}^{\alpha_{2}} \quad(i=1,2)$ and $\beta P^{\alpha_{1}}=\beta P^{\alpha_{2}}$. In the following section we search for possible phase transitions between the same types of phases, such as nematic-nematic and smectic-smectic demixing transitions, and we examine the possibility of nematic-smectic, nematic-columnar and smectic-columnar 
phase transitions. The extent of the fractionation and the structure of the stable phases are studied in detail.

\section{$3 \quad$ Results and discussion}

\subsection{Phase behaviour of monodisperse hard cylinders}

The phase behaviour of parallel cylinders has been studied thoroughly several times by Monte Carlo simulation methods [15,21,41] and Onsager type theories [23,24,39-40,42]. More recently a sophisticated density-functional theory, the Fundamental-Measure theory (FMT), has been applied also to this system [22,43]. Mulder [39] has shown that Onsager's second virial theory is capable to describe the nematic-smectic A (N-S) phase transition of parallel hard cylinders. It was proved that the N-S phase transition is of second order and that the transition density (scaled with particle volume) is independent of the length-to-breadth ratio $(L / D)$. The results of Onsager theory for the transition properties are presented in Fig. 1. The distance between neighbouring layers at the N-S transition is about 1.39 L. Regarding the columnar (C) phase we are only aware of some bifurcation analysis studies of the nematiccolumnar phase transition based on Onsager theory $[13,40]$. It was found that, within this theory, the nematic phase becomes unstable with respect to columnar density modulation well above the N-S phase transition, which means that the N-C phase transition cannot take place. Capitán et al. [22] proposed a FMT approximation for the system of parallel cylinders, obtaining the complete phase diagram. The $\mathrm{C}$ phase was found always to be metastable, the sequence of stable phases being N-S-K (crystal phase).

Even though the $\mathrm{C}$ phase is probably preempted by the $\mathrm{K}$ phase in the monodisperse fluid, we expect that this phase will be stabilized in the bidisperse system. Consequently, in what follows we discuss the formation of the columnar phase in the monodisperse fluid with a view to obtaining the limits of phase stability in the phase diagrams of the mixture. We 
proceed beyond the bifurcation analysis of Onsager theory by obtaining the full numerical solutions of the columnar equations [see Eq. (7)] for Fourier coefficients and wave number in the monodisperse case at a given packing fraction $\left(\eta=\rho v_{0}\right.$, where $v_{0}$ is the volume of the cylinder). Two columnar solutions are detected in the packing fraction range of $0.83<\eta$ $<0.945$ : one solution is a weakly ordered columnar phase, while the other corresponds to a strongly ordered columnar structure. The weakly ordered columnar phase has a larger lattice constant $(a)$ than the strongly ordered one. In accordance with the bifurcation analysis the weakly ordered columnar phase is less ordered with increasing density and becomes nematic at the N-C bifurcation packing fraction $\left(\eta_{b i f} \approx 0.945\right)$, while the strongly ordered columnar phase exists at the $\mathrm{N}-\mathrm{C}$ bifurcation and becomes more ordered with increasing packing fraction. The two columnar solutions at $\eta=0.9$ are depicted in Fig. 2, while the equations of state of the nematic and columnar branches are shown in Fig. 3. Note that the nematic and columnar curves of Fig. 3 resemble the equation of state of a typical first-order phase transition [44]. Because of the presence of a van der Waals-like loop in the equation of state the nematic-columnar phase transition would be first order, provided it existed as a stable phase transition. The calculated coexisting nematic and columnar packing fractions are 0.814 and 0.888 , respectively. These values are above the N-S transition packing fraction, so the nematic-columnar phase transition is unstable. It is worth noting that the columnar solution exists much below the nematic-columnar bifurcation packing fraction.

The next issue is the stability of the smectic phase with respect to columnar order. Our smectic and columnar free energy calculations indicate that the smectic phase becomes unstable with respect to columnar structure at unphysically high densities with an accompanying first order smectic-columnar phase transition. The coexistence packing fractions of Onsager theory (see Fig. 1) are unphysical because they are above the close packing limit of the cylinders. This discrepancy of Onsager theory is due to the fact that it is a 


\subsection{Phase behaviour of length-bidisperse hard cylinders}

Since the smectic A phase is the first in the rank which destroys the nematic order in the monodisperse system of hard cylinders, it is reasonable to examine first the stability of the nematic phase with respect to a smectic A modulation in the mixture of short and long cylinders. The simplest method to locate the borders of the nematic and smectic regimes is the nematic-smectic bifurcation analysis. In our formalism this is equivalent to finding the lowest density at which Eq. (10) still has nonzero solutions for the Fourier coefficients of the positional distribution functions. In this regard the composition $\left(x_{i}=N_{i} / N\right)$ and the length ratio of the system $\left(l=L_{2} / L_{1}\right)$ are free parameters in the calculations. The bifurcation analysis of Koda and Kimura [12] shows that the nematic phase can be destabilized either by a monolayered smectic phase $\left(S_{1}\right)$ or by a bilayered or microsegregated smectic one $\left(S_{2}\right)$. The length-bidispersity always destabilizes the smectic order with respect to the nematic phase because the characteristic smectic periods of the short and long rods do not fit. For example, in a short-rod-rich smectic phase the added long rods cannot accommodate into the layer 
structure of the short rods, but they have to occupy more than one layer. Depending on the length of the long rod the number of occupied layers can start from one and go up to infinity. To minimize the excluded volume between short and long rods the long rods occupy the optimum position in the layer. With monolayered or bilayered stacking it is possible to reduce the number of layers overlapping with a long rod by one. For example, a long rod which occupies $n$ layers in monolayered structure, can interact with only $n-1$ in a bilayered smectic structure. The resulting stable phase is the bilayered structure, because there is an entropy gain from the more efficient packing. The opposite situation can also happen that the stable monolayered structure has occupation number $n$-1, while the metastable bilayered structure has $n$. Koda and Kimura [12] have derived analytical equations for the structure of the smectic phase at the nematic-smectic bifurcation in the limit of pure phases $\left(x_{1} \rightarrow 0\right.$ and $\left.x_{1} \rightarrow 1\right)$. The regions of $S_{1}$ and $S_{2}$ phases alternate according to

$$
\begin{gathered}
(2 i-1) a_{N S}-L_{2} \leq L_{1} \leq 2 i a_{N S}-L_{2}, \\
0 \leq L_{1} \leq a_{N S}-L_{2} \text { and } 2 i a_{N S}-L_{2} \leq L_{1} \leq(2 i+1) a_{N S}-L_{2},
\end{gathered}
$$

$(i=1,2,3, \ldots)$ where the first equation gives the interval of $L_{2}$ for a stable $\mathrm{S}_{1}$ structure, while the second one is for the $\mathrm{S}_{2} . a_{N S}$ is the smectic period at the nematic-smectic bifurcation of the pure phase of component 2, which equals $1.39 L_{2}$. In Fig. 4 we present the results of bifurcation analysis for the structure, the packing fraction and the period of the smectic phase as a function of composition and length ratio. We can see from Fig. 4a that our results follow the prediction of Eq. (12), but it also gives further information about the stability of smectic structures for intermediate compositions. In accordance with Eq. (12) we find $S_{2}-S_{1}-S_{2}-S_{1}$ sequences for $0.2<l<1$ in the short-rod-rich regions, while systems with a majority of long rods show only the $S_{2}-S_{1}$ change. Figures $4 b$ and $4 c$ demonstrate the commensuration problem of the particles' lengths. Both the bifurcation packing fraction and the smectic period have abnormal behaviour at intermediate values of length ratio and composition. The packing 
fraction goes up to unphysical ranges in the vicinity of an equimolar mixture, while the smectic period shows increasing discontinuity with decreasing length ratios. The results clearly show the suppression of the smectic phase and the possible predominance of stable columnar structures, because the columnar phase does not suffer from the commensuration problems.

A more detailed insight is provided by the positional distribution functions $f_{i}(z)=\rho_{i}(z) / \rho_{i}$ of the possible smectic phases. Numerical solution of Eq. (10) results in three qualitatively different smectic structures: a monolayered (Figs. 5a) and a bilayered structure (Fig. 5b), and a two-in-one-layer ordering (Fig. 5c). We can see that the long rods are more ordered than the short ones in all three cases. This is due to the fact that the long rods constitute the majority species and they contribute with a higher excluded volume cost than the short ones. The shorter rods can move out more freely from the center of the layer without increasing the excluded volume cost. The conventional smectic phase $\left(\mathrm{S}_{1}\right)$ is mainly stable in weakly length-bidisperse systems $(l>0.6)$, but it can also take place in the strongly bidisperse case. In the special case of $L_{2}<0.39 L_{1}$, the short rods can accommodate in the interstitial region and the short rods are squeezed out from the layer of the long rods to make the packing of the short and long rods more efficient. The resulting bilayered smectic structure is shown in Fig. 5b. The two-in-one smectic ordering $\left(\mathrm{S}_{3}\right)$ is the most interesting (Fig. 5c), because it has not been predicted theoretically before. The first experimental evidence for the existence of a $S_{3}$ smectic structure is reported in the very recent study of Okashi et al [38]. It is found that the two-layer accommodation is possible in the length ratio interval of $0.34<l<0.59$. Note that the length ratio of Fig. $5 \mathrm{c}$ is located inside this interval. The existence of the $S_{3}$ structure is due to commensuration, because two layers of shorts rods can fit into one layer of long rods. Finally Fig. 5. proves that our model and the experimental 
system are in close relationship because the smectic structures of the binary mixture of short and long polysilanes are those shown in Fig. 5.

We have monitored how the structural change takes place in the smectic phase by changing the length ratio at a given pressure. We have chosen two compositions $(x=0.01$ and 0.99) very close to the monodisperse limits to avoid the stability problem of the smectic phase. Figure 6 demonstrates that $S_{1}$ and $S_{2}$ structures transform into each other continuously. In the long-rod-rich phase (Fig. 6a) the short rods go into the interstitial region with decreasing length ratio. At around $l=0.3$ the short rods form a nematic phase because they can stay everywhere with equal probability. This is an interesting structural behaviour because the long rods are in the smectic phase irrespectively of the value of the length ratio. In the shortrod-rich smectic phase we observe the same structural $S_{1}-S_{2}$ change in the distribution of long rods (see Fig. 6b). The only difference is that the period of the smectic phase is now shorter. To locate the border of $S_{1}-S_{2}\left(S_{2}-S_{1}\right)$ smectic transformations we have used the condition $f_{2}(z)=1\left(f_{1}(z)=1\right)$ in the solution of Eq. (10). The $S_{3}$ phase can only appear in long rod rich phases in the vicinity of $l=0.5$. In this region of length ratio the stable phases are the $S_{1}$ and $S_{3}$ structures. Since the transformation of the positional distribution function of the short rods from $S_{1}$ to $S_{3}$ is continuous, the second derivative of $f_{2}$ must be zero with respect to $z$ at $z=0$ and this helps to locate the border of $S_{1}-S_{3}$ structural change. For this reason we have used the condition $\left.\frac{d^{2} f_{2}}{d z^{2}}\right|_{z=0}=0$ to search for the $\mathrm{S}_{1}-\mathrm{S}_{3}$ transformation.

With the help of the nematic, smectic and columnar free energies we are in a position to construct the phase diagram of the system of short and long rods. Solving the phase equilibrium conditions we obtain the densities and compositions of the coexisting phases. The resulting phase diagrams are presented in the pressure-composition plane for the most important length ratios in Fig. 7. In the first system the lengths of the components are not far 
from each other. Mixing the short and long rods does not result in a substantial change in the nematic-smectic A second order phase transition. Owing to the difference in the lengths only weak destabilization of the smectic phase can be seen with respect to the nematic one. Interestingly, the smectic-columnar biphasic region moves into the direction of lower pressure by adding short (long) rods to the system of long (short) rods. This means that, contrary to the smectic phase, the columnar phase becomes more stable with decreasing length ratio. We can also observe weak fractionation between the coexisting phases. At around $x=0.4$ the coexisting smectic and columnar phases have the same composition, i.e. an azeotropic point bounds the stability region of the columnar phase. We can expect that the upper bound of the nematic and the lower bound of the columnar phase meet at lower length ratio. This happens slightly above $l=0.6$. We can see that a first order nematic-columnar phase transition takes place at $l=0.6$ in a narrow composition and pressure range. The nematic-columnar phase transition is terminated by a critical endpoint where it meets the N-S critical line. At this point the critical phase coexists with the columnar phase. Interestingly, a pressure-induced $\mathrm{S}_{1}-\mathrm{S}_{2}$ structural change takes place on the short-rod-rich side. This is due to the fact that the smectic layers get closer to each other with increasing pressure and the long rods enter into the two neighbouring layers. By moving into the interstitial region the long rods interact with only two layers; the packing entropy gain gives rise to the structural change in the smectic phase. In accordance with our results the system of short and long polysilanes shows only $\mathrm{S}_{1}$ ordering both in short and long rod rich phases in the range of $0.59<k<1$ [38]. At $l=0.5$ the smectic phase is microsegregated on the short rod side in accordance with Eq. (12), the nematic-columnar phase coexistence region widens, and a small region where the nematicsmectic phase transition is of first order (with a corresponding three phase point) is detected close to a composition of $50 \%$. In addition a $\mathrm{S}_{3}$ smectic structure occurs in a finite range of composition, which is rich in long rods. The interesting lower pressure border of the $S_{3}$ 
structure shows that the density of the short rods must exceed a minimum value to produce double layering. At the lower length ratio of $l=0.4$ we obtained the same phase diagram as for $l=0.5$, except for the $S_{1}-S_{2}$ change and for the fact that the nematic-smectic phase transition is of second order. We can also observe that the fractionation of the N-C phase transition gradually increases with decreasing length ratio. The phase behaviour of the experimental system resembles closely the phase behaviour of the hard cylinder mixture, because the $\mathrm{S}_{3}$ $\left(\mathrm{S}_{2}\right)$ structure is stable at compositions rich in long (short) rods in the region of $0.34<l<0.59$ and the short rod rich smectic phase is microsegregated [38]. The phase diagram of $l=0.3$ shows that a $\mathrm{S}_{2}-\mathrm{S}_{1}$ structural change takes place upon compression. In this case the short rods can accommodate in the interstitial region, but the compression moves the layers closer to each other and the short rods penetrate into the two layers. To avoid the double penetration the short rods move inside the layer and the system forms a monolayered smectic phase. Note that no $\mathrm{S}_{3}$ ordering takes place at $l=0.3$, which agrees with the experimental observations [38]. Another interesting result is that the lower boundary of the columnar phase starts to move up, i.e. the columnar phase destabilizes due to the efficient $S_{2}$ stacking. In addition the N-C phase boundary becomes very wide. At the lowest value of length ratio $(l=0.15)$, we can clearly see the destabilization effect of the $S_{2}$ stacking on the columnar phase. The stability region of the smectic phase is very wide, while that of columnar moves up and becomes very narrow. The very short and the long rods do not want to form columns, but they segregate very strongly at high pressures: the short-rod-rich nematic phase is in coexistence with a microsegregated smectic phase. We have also examined the possibility of a nematic-nematic demixing transition, but it is always located in the biphasic region of the $\mathrm{N}-\mathrm{S}_{2}$ phase boundary. Our attempts to find a stable N-N phase transition at very low length ratios has not been successful, because the $\mathrm{N}-\mathrm{S}_{2}$ transition becomes wider and moves down into the direction of lower pressure. 
In summary, the occurrence of a stable columnar phase in the intermediate length ratio range is the result of the subtle interplay between translational, packing and mixing entropies. The short and long rods cannot accommodate together into the stacks of layers without high packing entropy loss. In addition the demixing of the smectic phase into phases rich in short and long rods is accompanied by high mixing entropy cost. The existence of two-in-one smectic $\left(\mathrm{S}_{3}\right)$ ordering in the vicinity of $l=0.5$ is due to the efficient packing of the short rods in the smectic layers of the long rods.

\section{Conclusions}

We have examined the phase behaviour of the binary mixture of short and long parallel hard rods as a function of length ratio using Onsager's second virial theory. One- and two-dimensional Fourier expansions have been applied to represent the smectic and columnar phases, respectively. We have found that the stability regions of the nematic, smectic and columnar phases are very sensitive to the length ratio. The nematic phase can be stabilized by length bidispersity because either the short or the long rods do not fit into the $1 \mathrm{D}$ periodic structure of the smectic phase rich in long rods or rich in short rods. This commensuration problem is very strong in the vicinity of equimolar composition where the short components are trying to create a $1 \mathrm{D}$ periodic structure with a short smectic period, while the long rods prefer the formation of a smectic phase with long smectic period. A further consequence of the commensuration problem is the stabilization of the columnar with respect to the smectic phase at high pressures (densities). With decreasing length ratio the regime of smectic stability shrinks, while those of the nematic and columnar phases become more extended. At $l=0.6$ the nematic and columnar regions meet and a first-order nematic-columnar phase transition is stabilized. The region of the nematic-columnar phase transition widens and it becomes more fractionated with decreasing length ratio. However, below $l<0.4$ the tendency 
changes because the rods of the short component can fit into the interlayer region of the neighbouring layers and a microsegregated (bilayered) smectic phase may form. Due to the efficient packing of the bilayered smectic structure and the gain in the translational entropy, the region of the smectic phase widens, while the columnar phase is suppressed and shifted into the direction of higher pressures. Therefore the stability of the columnar phase is limited by lower and upper bounds in the length ratio. To stabilize the columnar phase the best systems are those where the long rods are 2-3 times longer than the short ones. No nematicnematic and smectic-smectic demixing transitions are found, since the strong nematic-smectic and nematic-columnar fractionations preempt the demixing transitions. We have shown that our binary mixture of short and long rods and that made of real polysilane molecules have the same types of smectic phases. In addition, the smectic phases in systems rich in short and long rods show the same structural changes with decreasing length ratio. Qualitative tests between theory and experiment to check the stability of nematic and columnar phases are not possible at the moment, because the objective of the study of Okoshi et al. [38] has been only the smectic ordering.

In conclusion our study shows that the phase diagram of the short and long rods system can be very complex due to commensuration effects of the particle lengths. It remains an open question whether the orientational entropy contributions change the topology of the phase diagrams in the nematic, smectic and columnar regimes. We hope that our work provides a useful guide for future experimental works on rod-like mixtures exhibiting spatially ordered mesophases such as the recently synthesized binary mixture of helical polysilanes [38]. 


\section{Acknowledgements}

We are grateful to Giorgio Cinacchi for useful discussions and for bringing our attention to the work of Okoshi et al. and to the $S_{3}$ smectic phase. SV and FV would like to thank the financial support of the Austrian-Hungarian Action Foundation (grant number: 73 öu1). Support from the Hungarian-Spanish (HH2006-0005) and Austrian-Spanish (HU20060006) Integrated Actions Programmes, and grants S-0505/ESP-0299 from the Comunidad Autónoma de Madrid, and FIS2007-65869-C03-01, FIS2008-05865-C02-01, and FIS200805865-C02-02 from the Ministerio de Educación y Ciencia of Spain, are also acknowledged.

\section{References}

[1] H. N. W. Lekkerkerker, Ph. Coulon, R. van der Haegen, and R. Deblieck, J. Chem. Phys. 80, 3427 (1984).

[2] G. J. Vroege and H. N. W. Lekkerkerker, J. Phys. Chem. 97, 3601 (1993).

[3] R. van Roij, B. Mulder, and M. Dijkstra, Physica A 261, 374 (1998).

[4] S. Varga and I. Szalai, Phys. Chem. Chem. Phys. 2, 1955 (2000).

[5] K. R. Purdy, S. Varga, A. Galindo, G. Jackson, and S. Fraden, Phys. Rev. Lett. 94, 057801 (2005).

[6] G. Cinacchi, E. Velasco and L. Mederos, J. Phys.: Conden. Matter 16, S2003 (2004).

[7] G. Cinacchi, L. Mederos and E. Velasco, J. Chem. Phys. 121, 3854 (2004); Y. Martínez-Ratón, E. Velasco and L. Mederos, J. Chem. Phys. 123, 104906 (2005).

[8] T. J. Sluckin, Liq. Cryst. 6, 111 (1989).

[9] A. Stroobants, Phys. Rev. Lett. 69, 2388 (1992).

[10] A. Stroobants, J. Phys.: Condens. Matt. 6, A285 (1994).

[11] S-M. Cui and Z. Y. Chen, Phys. Rev. E. 50, 3747 (1994). 
[12] T. Koda and H. Kimura, J. Phys. Soc. Japan 63, 984 (1994).

[13] R. P. Sear and G. Jackson, J. Chem. Phys. 102, 2622 (1995).

[14] T. Koda, M. Numajiri, and S. Ikeda, J. Phys. Soc. Japan 65, 3551 (1996).

[15] T. Koda and S. Ikeda, Mol. Cryst. Liq. Cryst. 318, 101 (1998).

[16] Z. Dogic, D. Frenkel, S. Fraden, Phys. Rev. E 62, 3925 (2000).

[17] T. Koda, A. Nishioka, and S. Ikeda, J. Phys.: Condens. Matt. 17, S2875 (2005).

[18] F. J. Vesely, Mol. Phys. 103, 679 (2005).

[19] D. Frenkel, Liquid Crystals 5, 929 (1989).

[20] D. Frenkel, Mol. Phys. 60, 1 (1987).

[21] D. Frenkel, H. N. W. Lekkerkerker, and A. Stroobants, Nature 332, 822 (1988).

[22] J.A. Capitán, Y. Martínez-Ratón and J.A. Cuesta, J. Chem. Phys. 128, 194901 (2008).

[23] A. Mori and H. Kimura, J. Phys. Soc. Japan 61, 2703 (1992).

[24] H. Xu, H.N.W. Lekkerkerker and M. Baus, Europhysics Lett. 17, 163 (1992).

[25] A. M. Bohle, R. Holyst and T. Vilgis, Phys. Rev. Lett. 76, 1396 (1996).

[26] R. van Roij and B. Mulder, Phys. Rev. E 54, 6430 (1996).

[27] M. A. Bates and D. Frenkel J. Chem. Phys. 109, 6193 (1998).

[28] Y. Martínez-Ratón and J.A. Cuesta, Mol. Phys. 107, 415 (2009).

[29] G. J. Vroege and H. N. W. Lekkerkerker, Rep. Prog. Phys. 55, 1241 (1992).

[30] P. A. Buining H. N. W. Lekkerkerker, J. Phys. Chem. 97, 11510 (1993).

[31] M. Adams, Z. Dogic, S. L. Keller, and S. Fraden, Nature 393, 349 (1998).

[32] P. K. Rai, R.A. Pinnick, A. N. G. Parra-Vasquez, et al., J. Am. Chem. Soc. 128, 591 (2006).

[33] S. E. Moulton SE, M. Maugey, P. Poulin, et al., J. Am. Chem. Soc. 129, 9452 (2007).

[34] J.P.F Lagerwall and G. Scalia, J. Material. Chem. 18, 2890 (2008).

[35] H. H. Wensink and G.J. Vroege, Phys. Rev. E 65, 031716 (2002). 
[36] A. Speranza and P. Sollich, J. Chem. Phys. 117, 5421 (2002).

[37] S. Varga, A. Gábor, E. Velasco, L. Mederos and F. J. Vesely, Mol. Phys. 106, 1939 (2008).

[38] K. Okoshi, A. Suzuki, M. Tokita, M. Fujiki and J. Watanabe, Macromolecules 42, 3443 (2009).

[39] B. Mulder, Phys. Rev. A 35, 3095 (1987).

[40] D. Frenkel, Freezing and Glass Transitions, Les Houches Session, edited by J. P. Hansen et al. (North-Holland, Amsterdam 1991).

[41] T. Koda and S. Ikeda, J. Chem. Phys. 116, 5825 (2002).

[42] Y. Martínez-Ratón and E. Velasco, J. Chem. Phys. 129, 054907 (2008).

[43] Y. Martínez-Ratón, J.A. Capitán and J.A. Cuesta, Phys. Rev. E 77, 051205 (2008).

[44] R. F. Kayser and H. J. Raveche, Phys. Rev. A. 17, 2067 (1978). 


\section{Figures}

Figure 1. Phase sequences of the monodisperse system of parallel hard cylinders. The solid phase is not included in the model.

Figure 2. Positional distribution function of a weakly ordered (a) and a strongly ordered columnar (b) phase at $\eta=0.9$. The lattice constant of the columnar phase: (a) $a^{*}=1.41$, (b) $a^{*}=1.39$

Figure 3. Columnar and nematic solutions in the pressure-packing fraction plane (reduced pressure: $P^{*}=\beta P v_{0}$, packing fraction: $\eta=\rho v_{0}$ ) for the pure system of parallel hard cylinders. Dashed line connecting the filled squares shows the coexisting nematic and columnar phases. Filled diamond symbol locates the nematic-columnar bifurcation point.

Figure 4. Nematic-smectic bifurcation of the binary mixture of short and long cylinders. a) Structure of the smectic phases in the length ratio-composition plane. $\sigma=1$ means microsegregated (bilayered) smectic phase $\left(S_{2}\right)$, while $\sigma=0$ denotes monolayered smectic phase $\left(S_{1}\right)$ b) Packing fraction $(\eta)$ of nematic-smectic bifurcation in the length ratiocomposition plane. c) Smectic period $\left(a^{*}=a / L_{1}\right)$ in the length ratio-composition plane. $x$ is the mole fraction of long rods.

Figure 5. Possible smectic phases of the binary mixture of short and long cylinders. a) Smectic phase where both components are in phase $\left(\mathrm{S}_{1}\right)$. b) Microsegregated smectic phase where the distributions of the components are out of phase $\left(S_{2}\right)$. c) Smectic phase where the 
components are in phase and the short rods forms two layers inside the layer of long rods $\left(\mathrm{S}_{3}\right)$ For all cases the values of the mole fraction of long rods and the reduced pressure are 0.6 and 4, respectively. The length ratios are 0.65 (a), 0.3 (b) and 0.5 (c).

Figure 6. Structural change in the smectic phase as a function of length ratio in long rod rich a) and short rod rich phases b). The composition is $x=0.99$ in a), while it is 0.01 in b). The value of the reduced pressure is 5 for all cases.

Figure 7. Phase diagram of the binary mixture of short and long cylinders in pressurecomposition plane. The values of the length ratio $\left(l=L_{2} / L_{1}\right): 0.65$ (a), 0.6 (b), 0.5 (c), 0.4 (d), 0.3 (e) and 0.15 (f). The horizontal short dashed lines connect either the nematic-smectic critical endpoint with the coexisting columnar phase or show the nematic-smectic-columnar three phase coexistences. In Fig. b) and e) the short dashed line connects also the terminal point of $S_{1}-S_{2}$ structural change with the coexisting columnar phase. The border of $S_{1-} S_{2}, S_{2}-$ $\mathrm{S}_{1}$ and $\mathrm{S}_{1}-\mathrm{S}_{3}$ structural transformations are denoted as a dashed curve. The long dashed curve is the boundary of the unstable nematic-nematic demixing transition. We use the following notations: N-nematic, $\mathrm{S}_{1}$-monolayered smectic, $\mathrm{S}_{2}$-bilayered smectic, $\mathrm{S}_{3}$-two-in-one smectic and C-columnar phase. $x$ is the mole fraction of long rods. 


\begin{tabular}{c|c|c|c} 
Nematic & Smectic & S+C & Columnar \\
\hline & & & \\
$\eta_{N-S}=0.575$ & $\eta_{S}=1.393$ & $\eta_{C}=1.518$ \\
$q_{S} L=4.494$ & $q_{S} L=4.972$ & $q_{C} D=4.795$ \\
$\left.\beta P v_{0}\right|_{N-S}=1.9$ & \multicolumn{2}{c}{$\left.\beta P v_{0}\right|_{S-C}=6.654$}
\end{tabular}

Figure 1. 


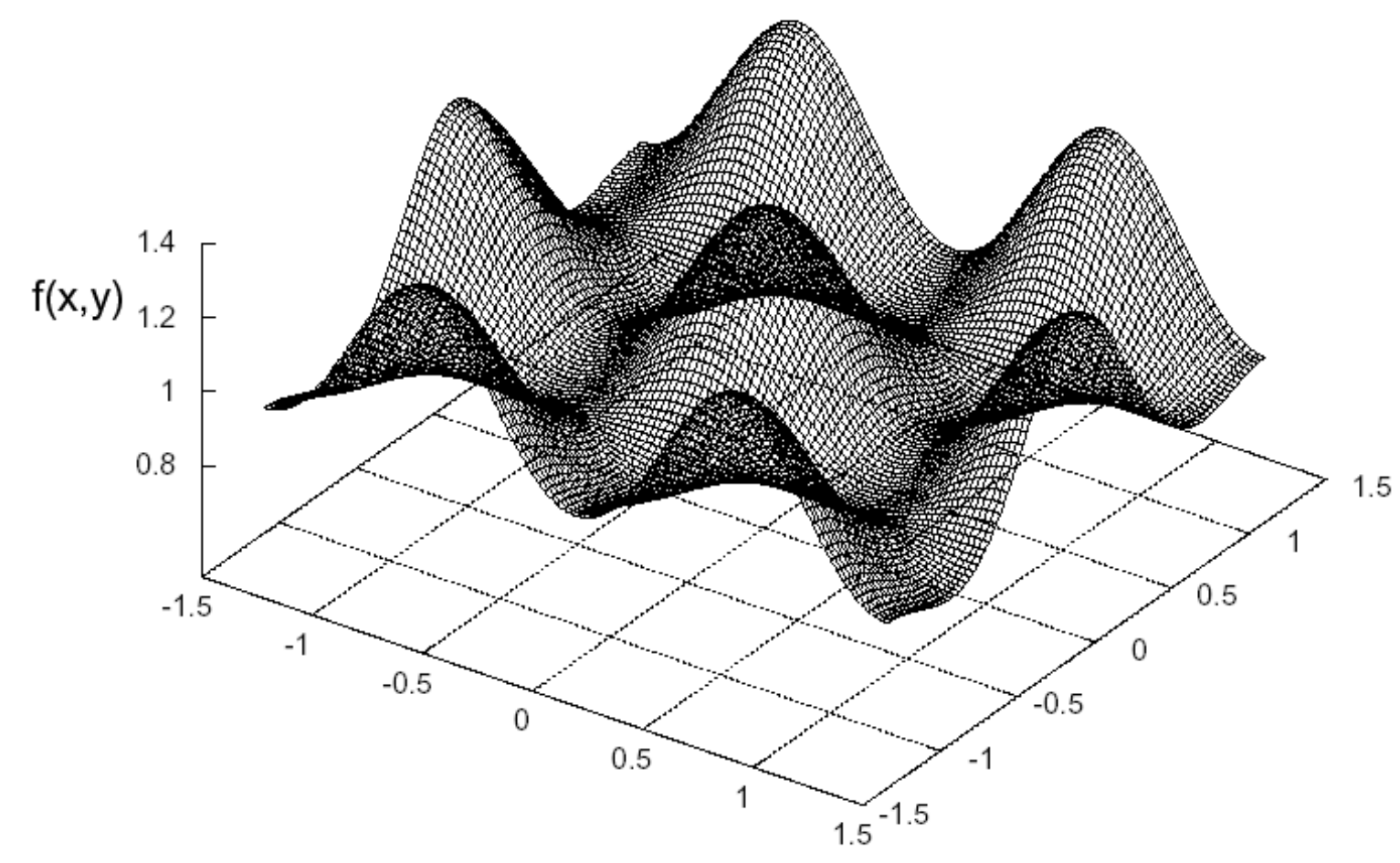

Figure 2 a)

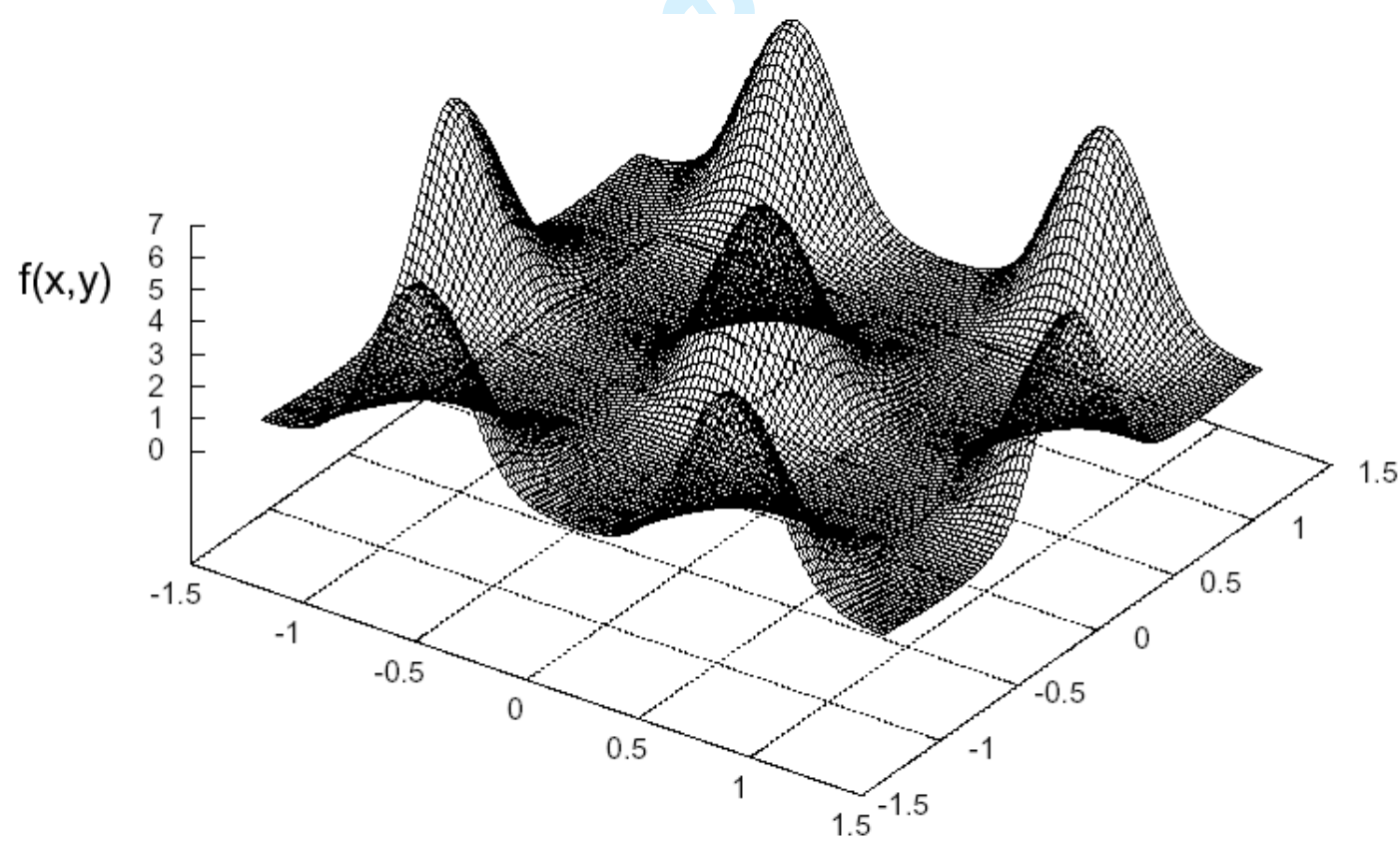

Figure $2 b$ ) 


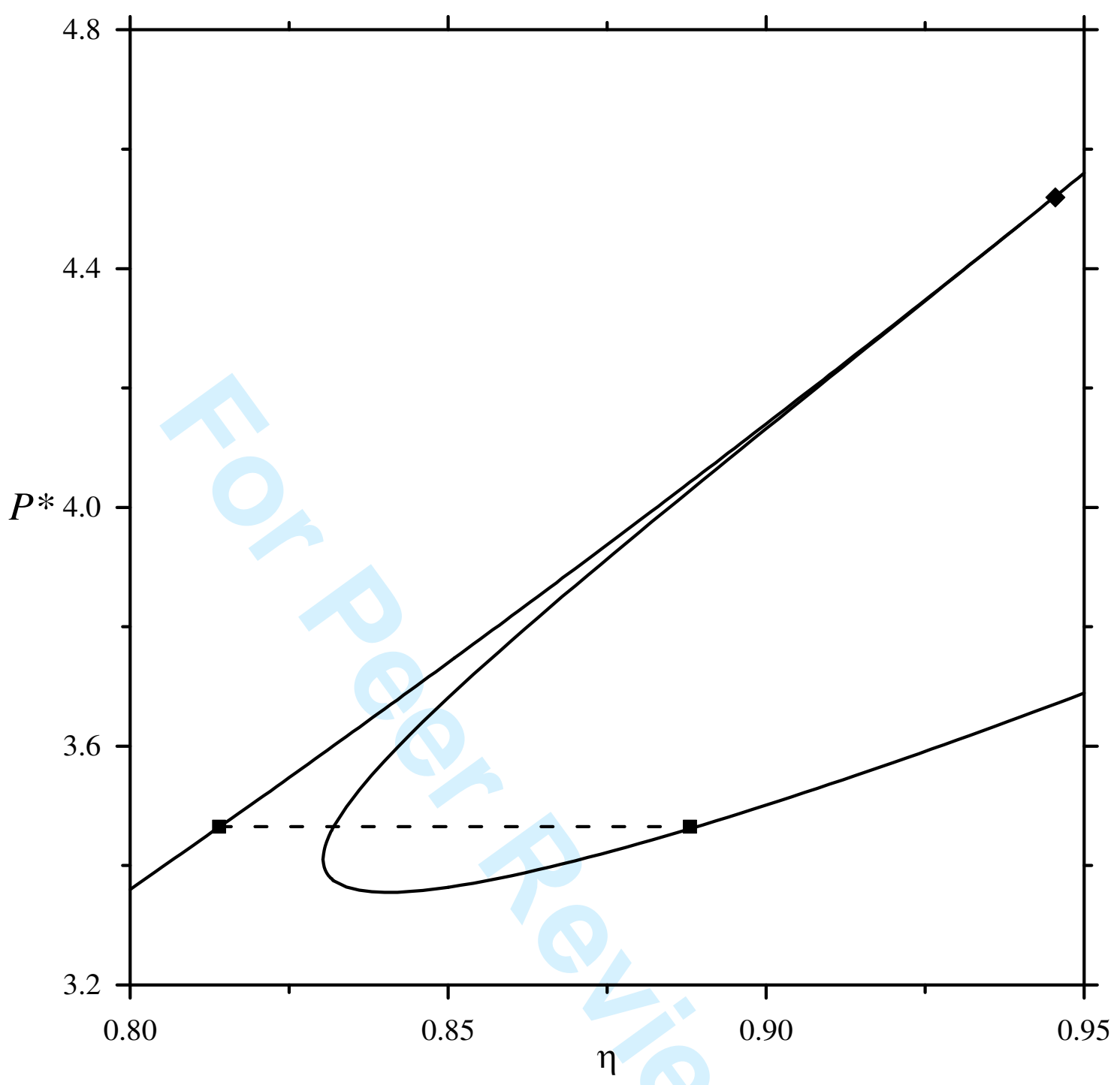

Figure 3. 


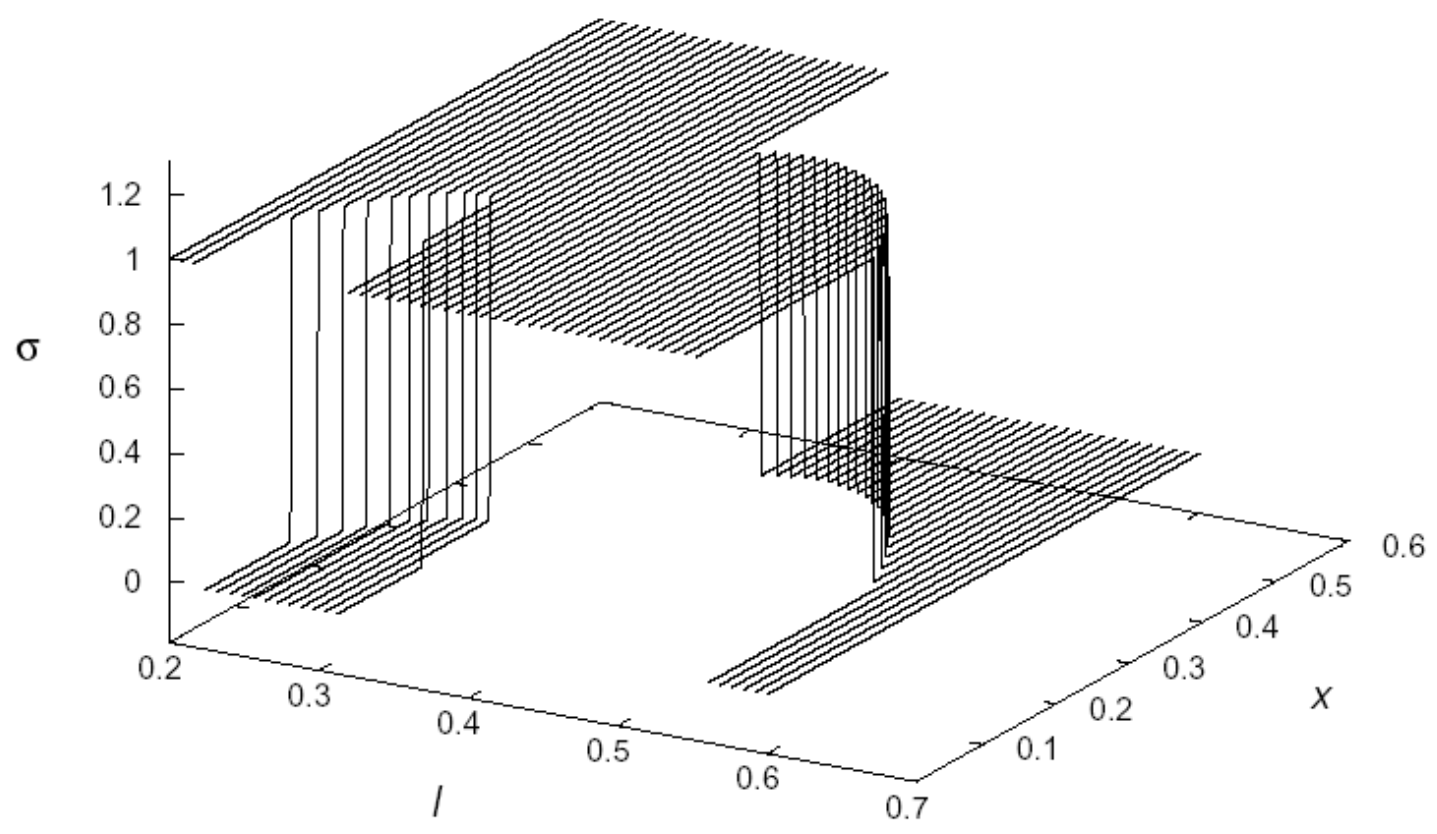

Figure 4 a)

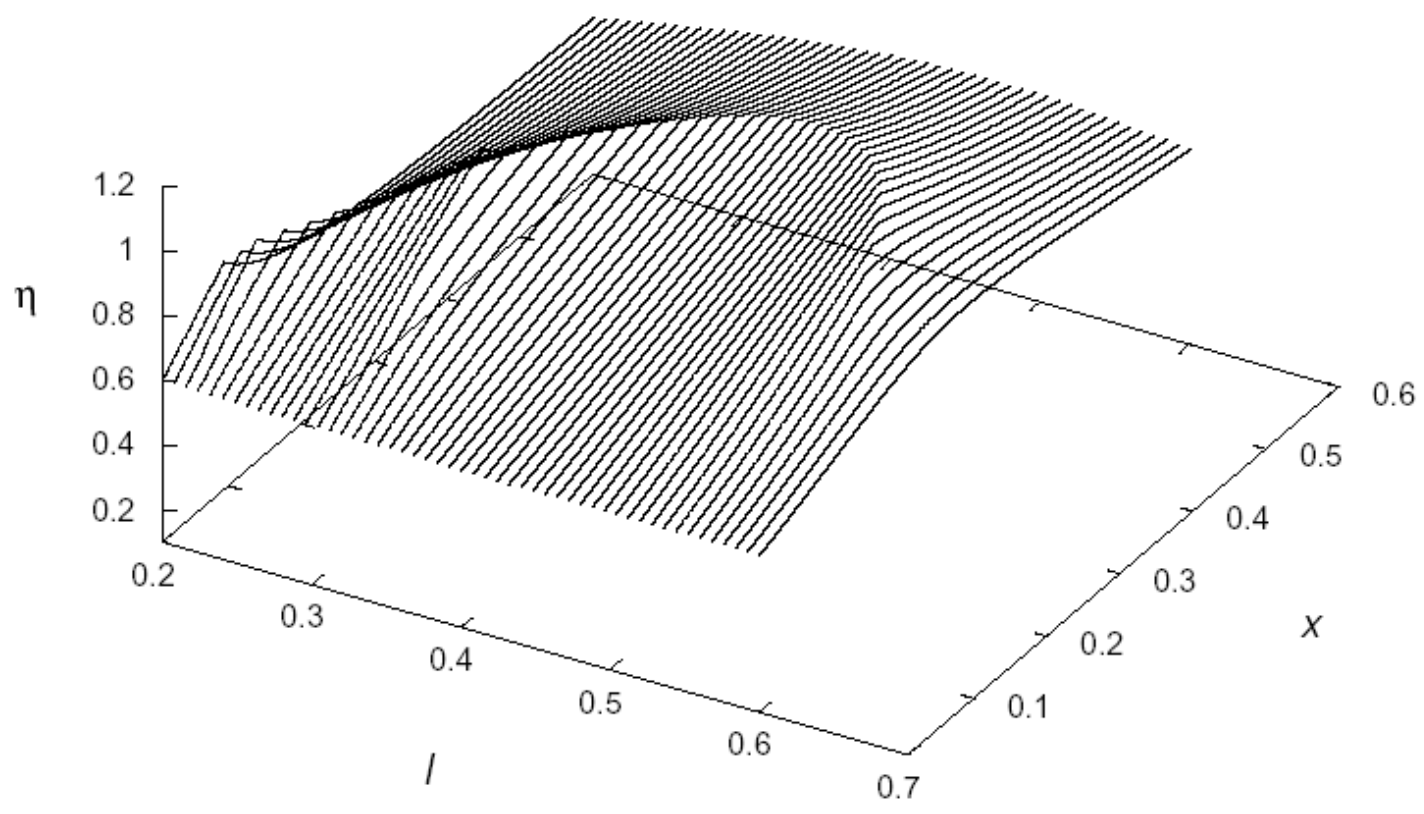

Figure $4 \mathrm{~b}$ ) 


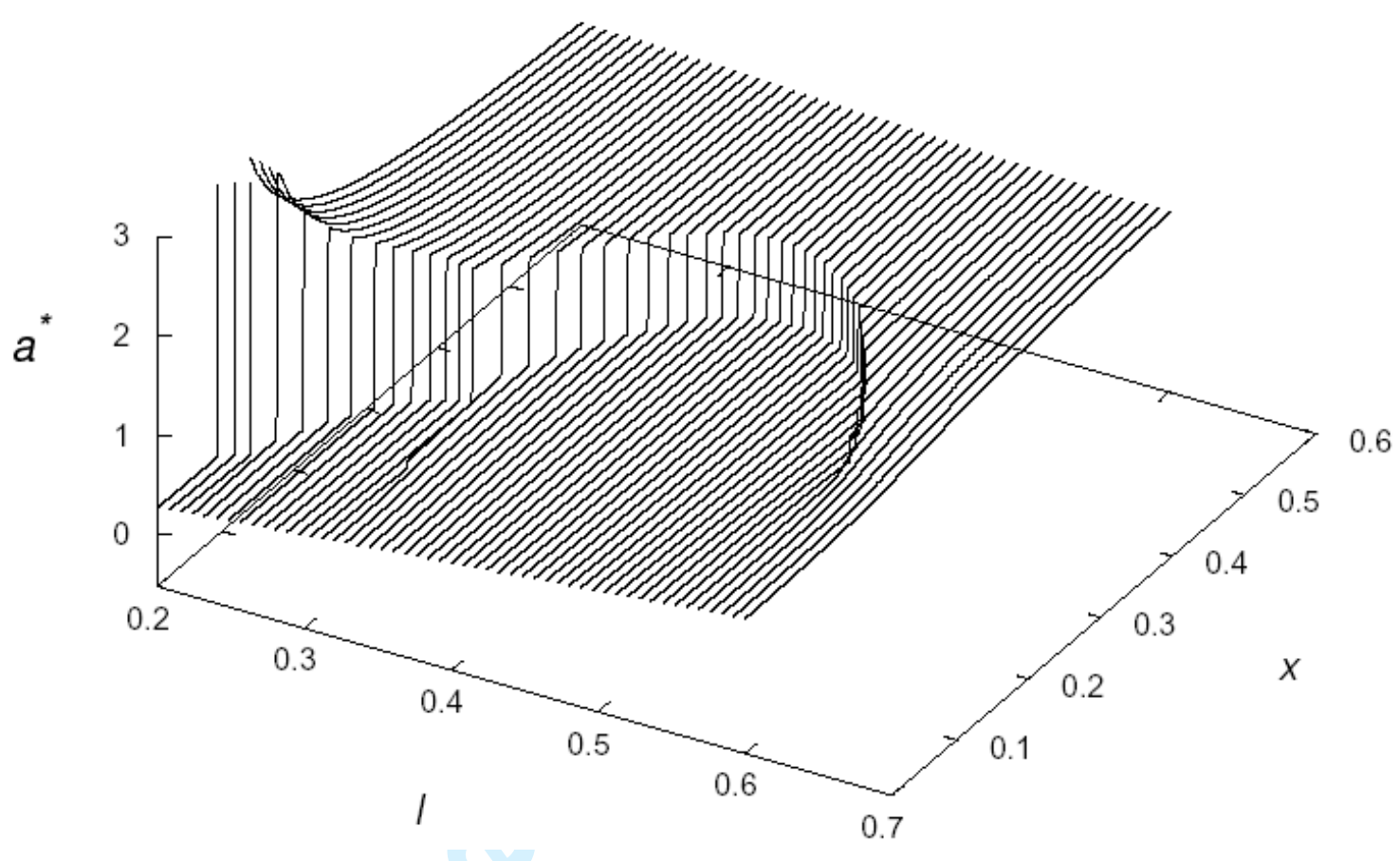

Figure $4 \mathrm{c}$ ) 

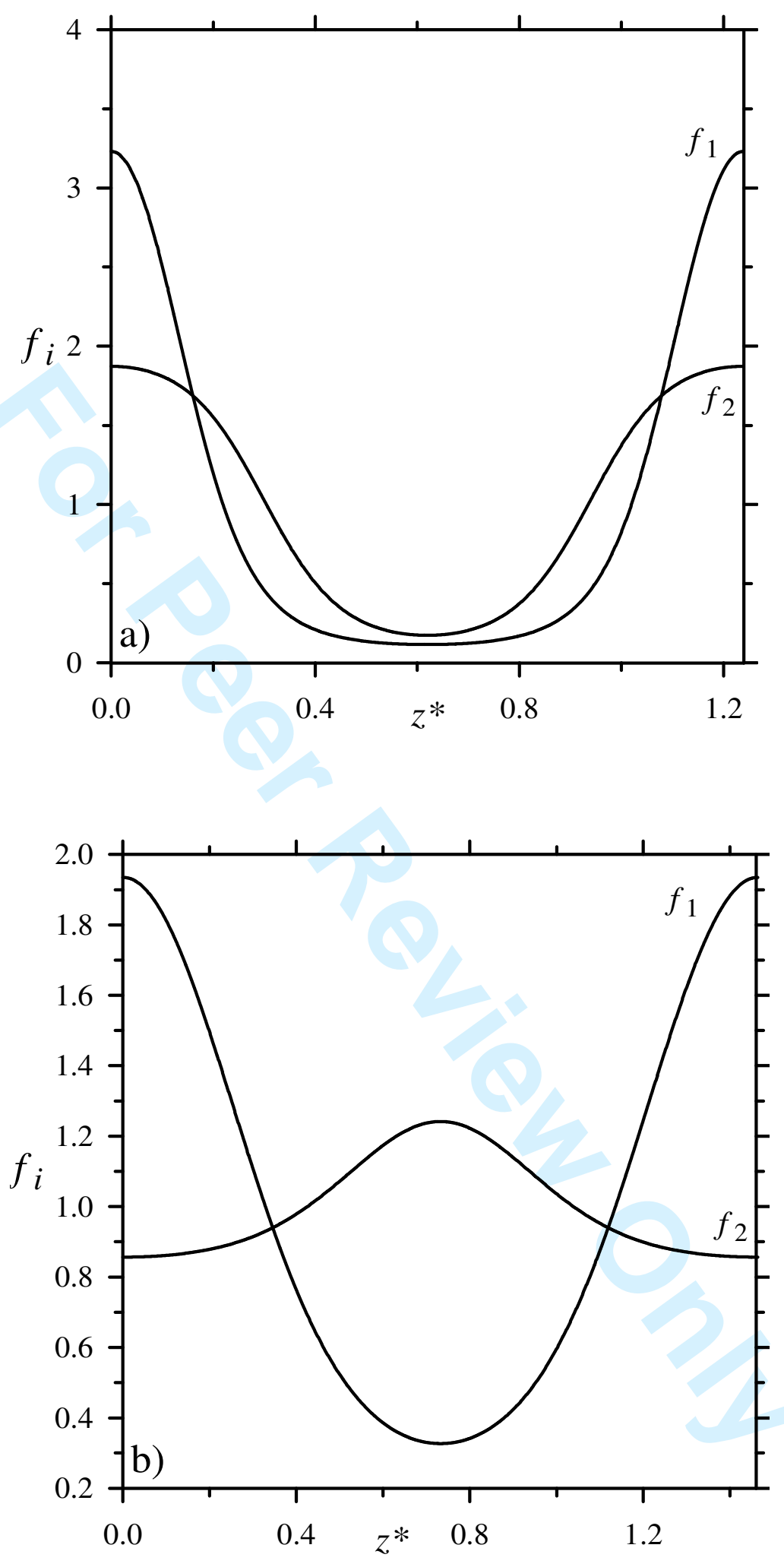

Figure 5 a) and b) 


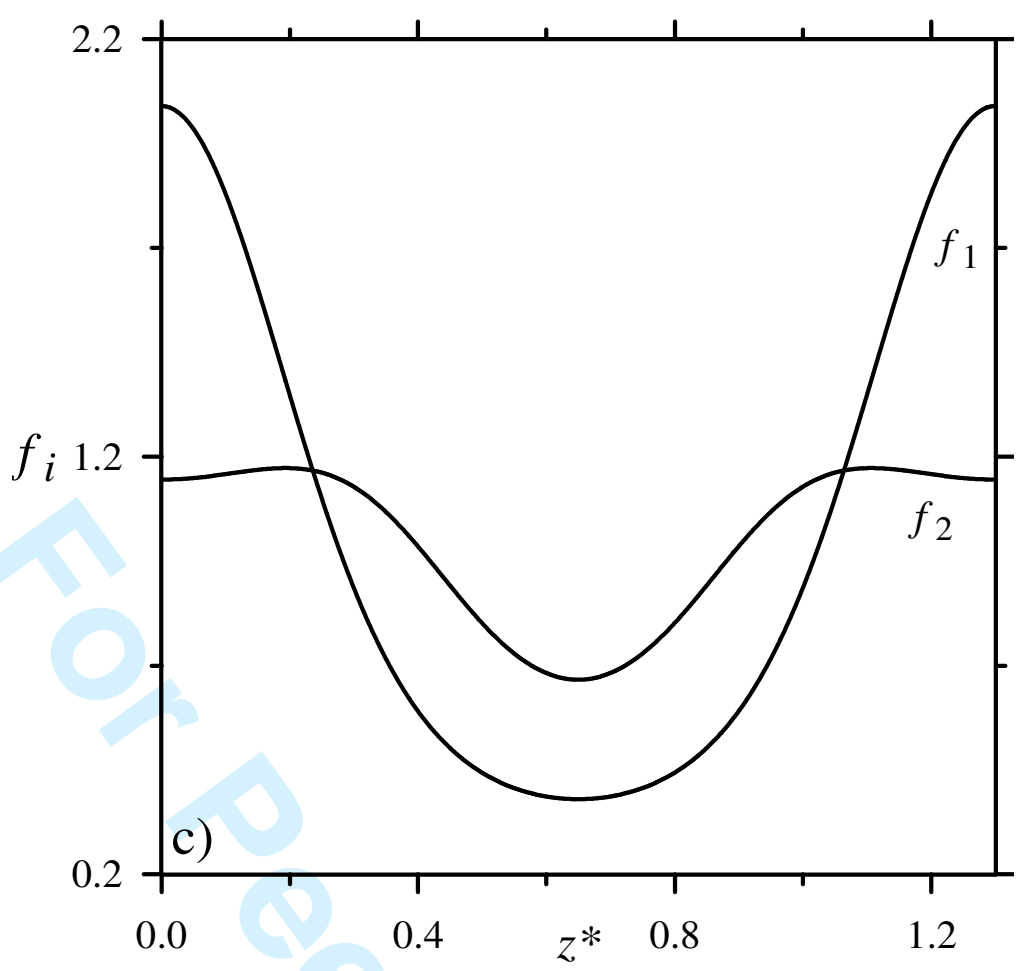

Figure 5 c) 


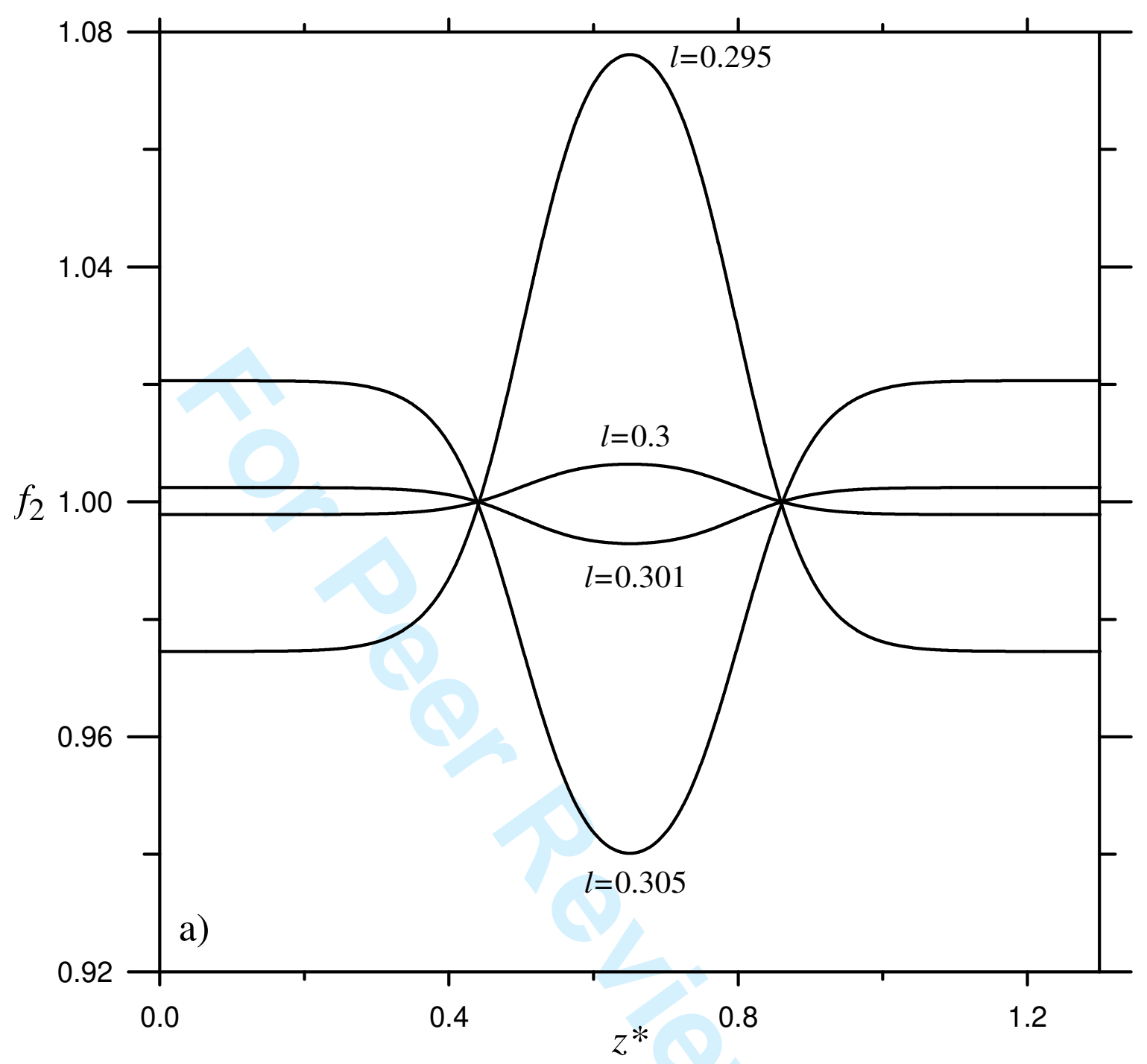

Figure 6 a) 


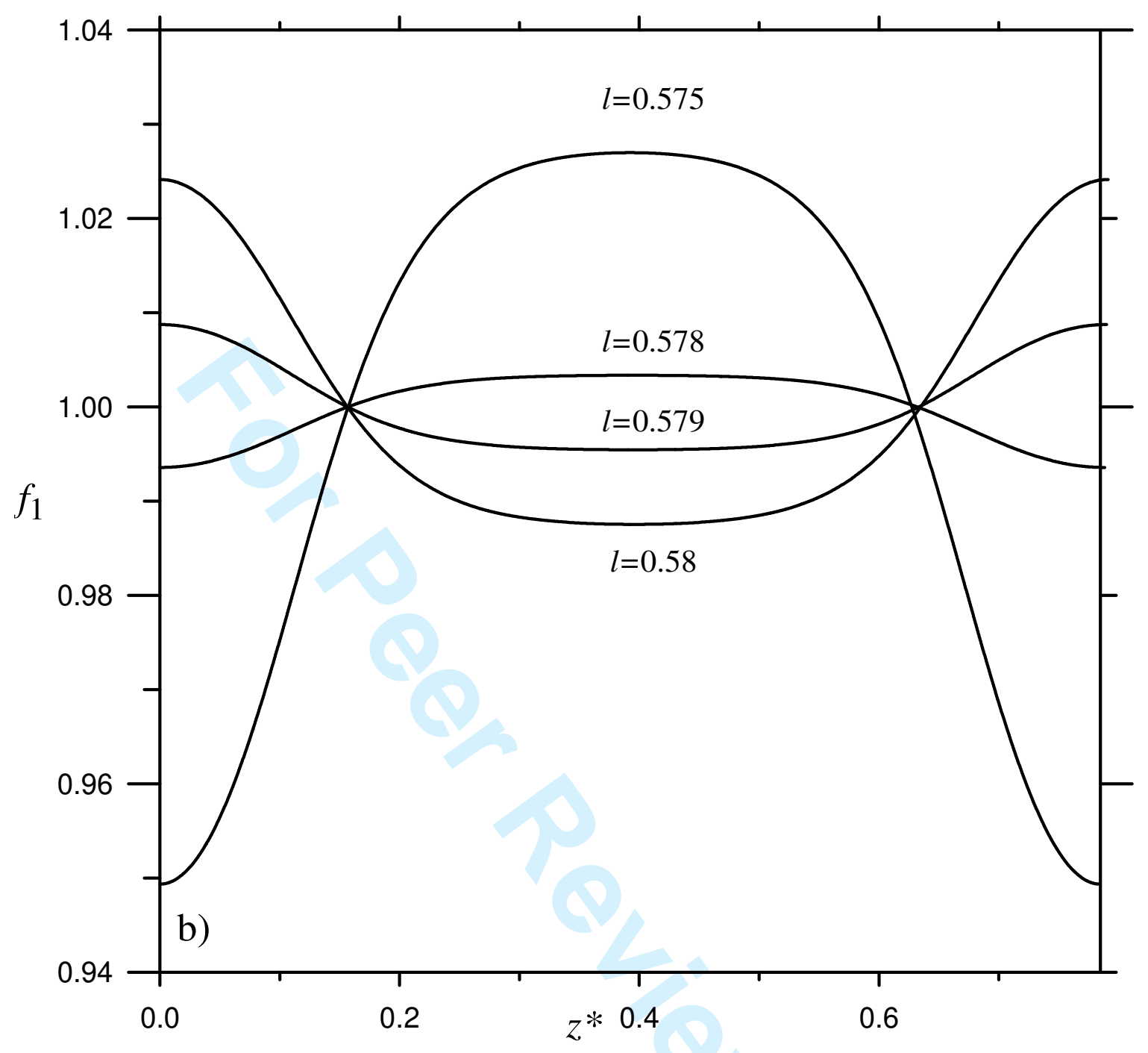

Figure 6 b) 


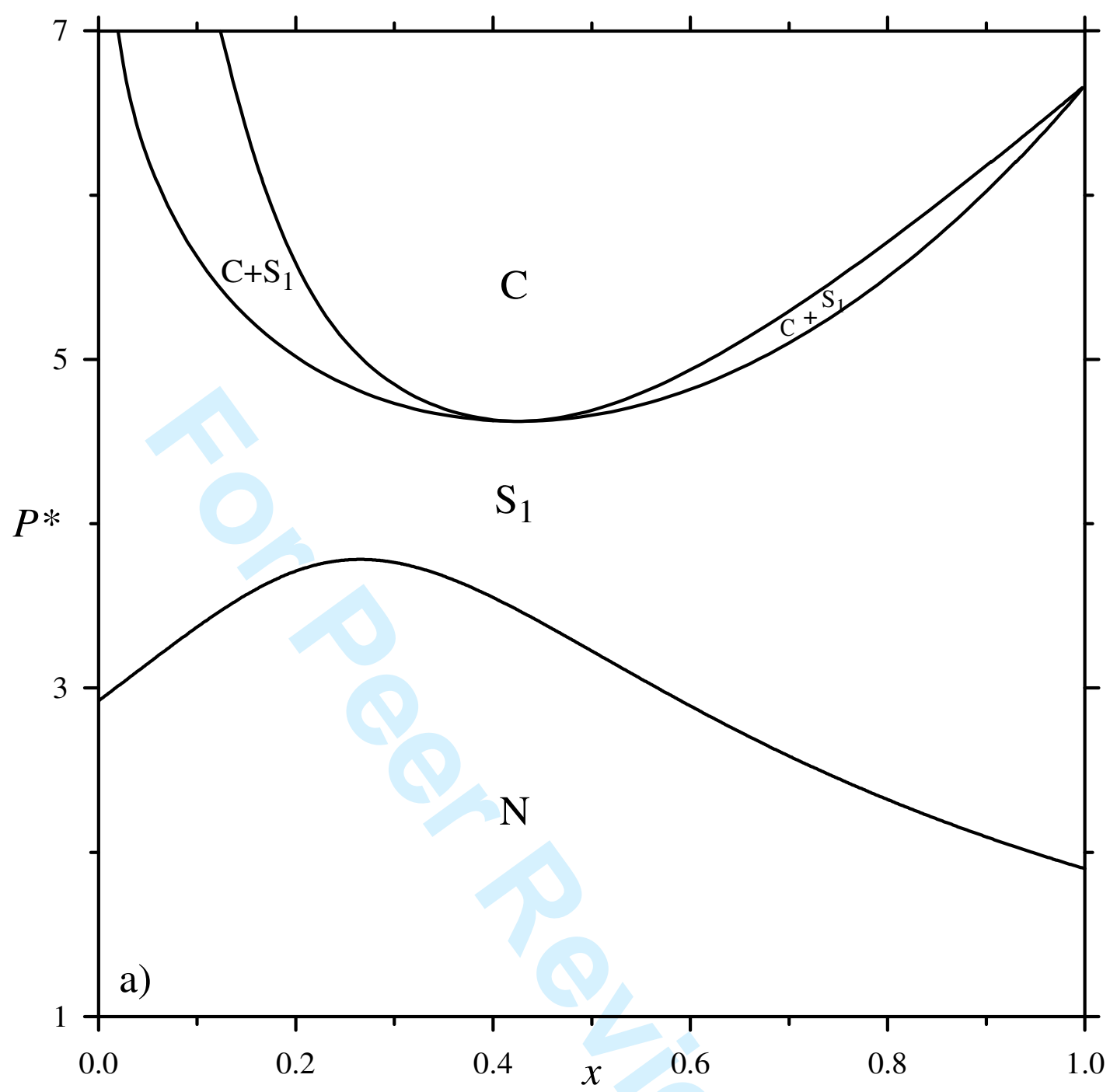

Figure 7 a) 


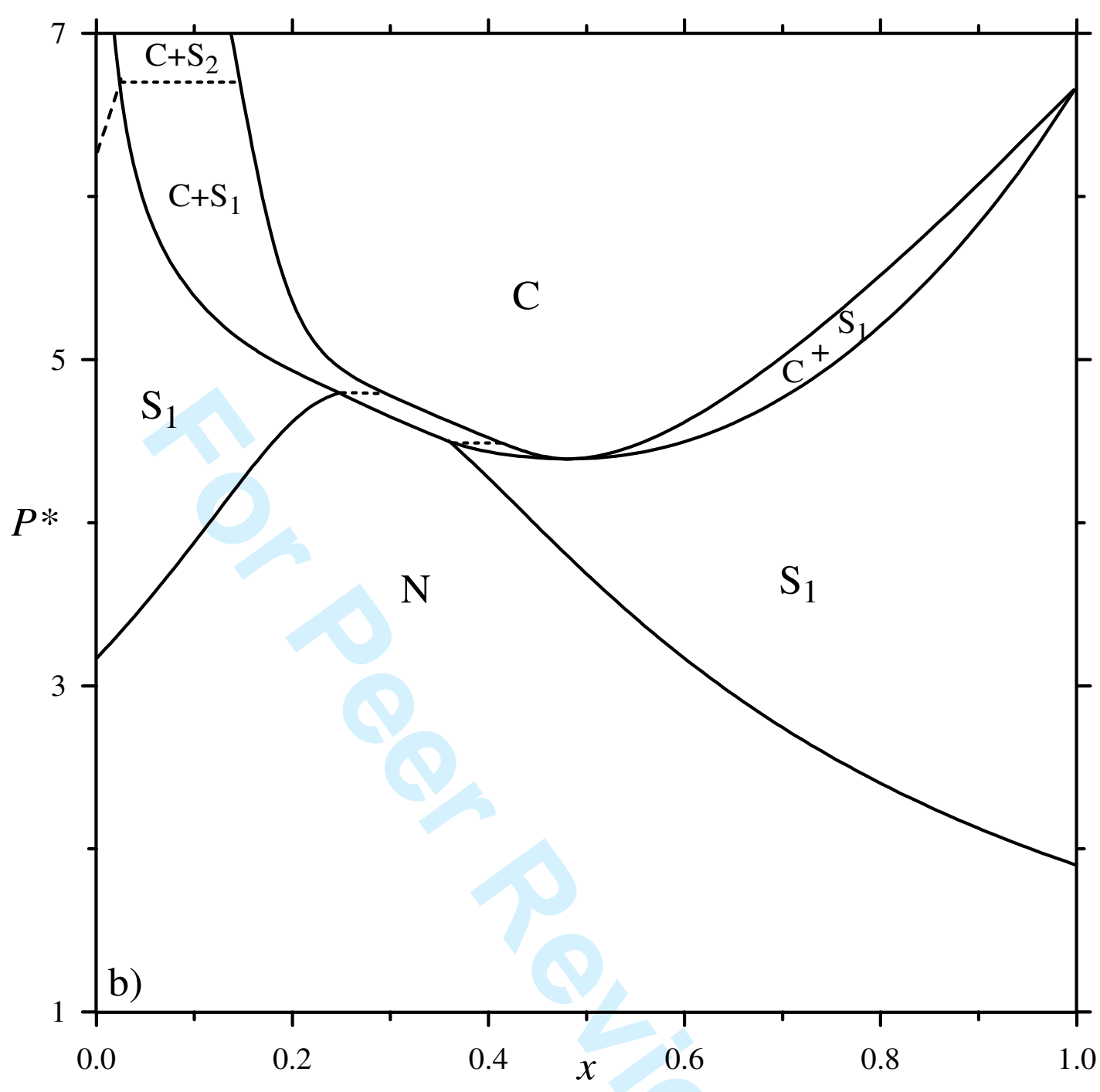

Figure 7 b) 


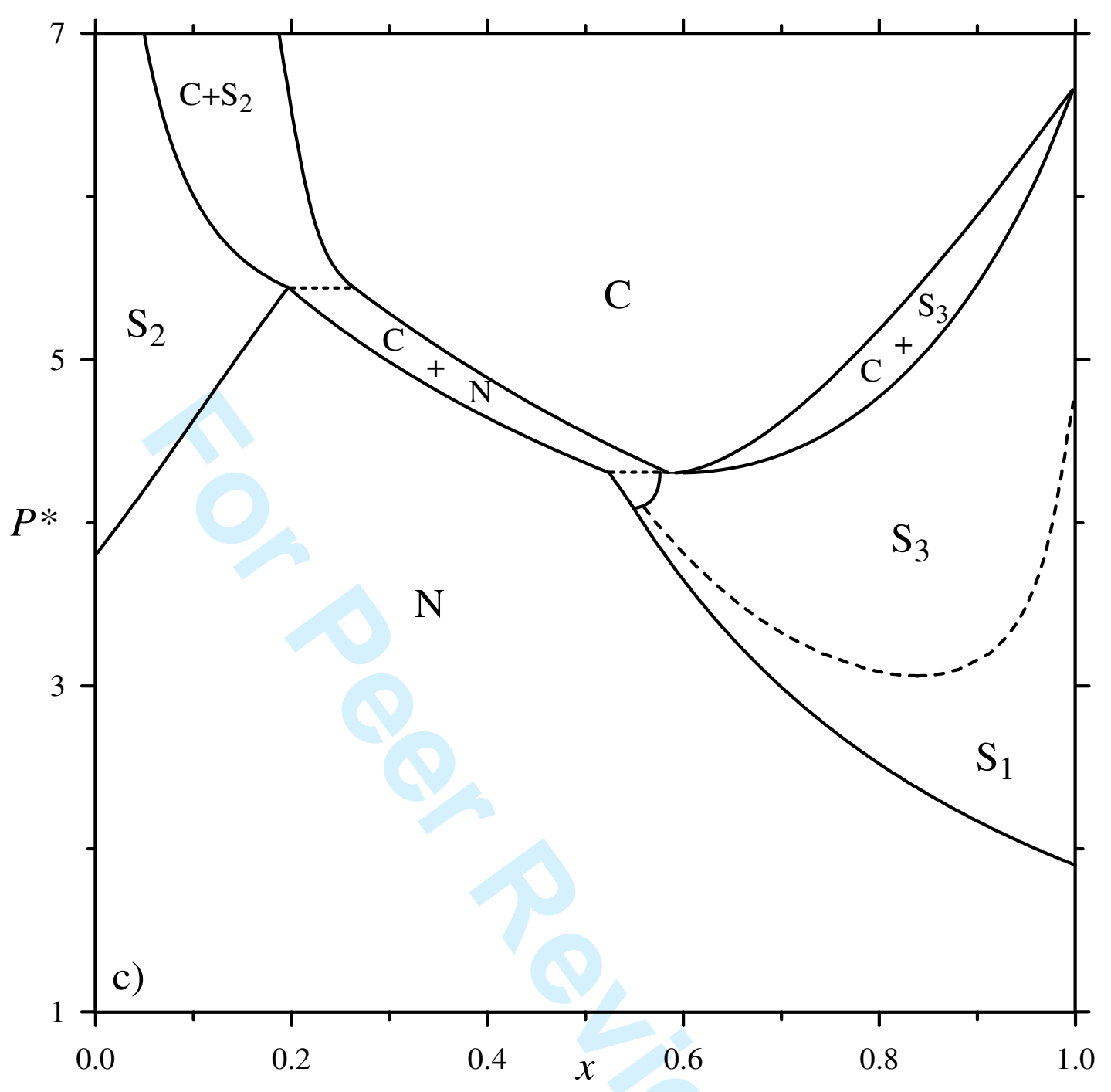

Figure 7 c) 


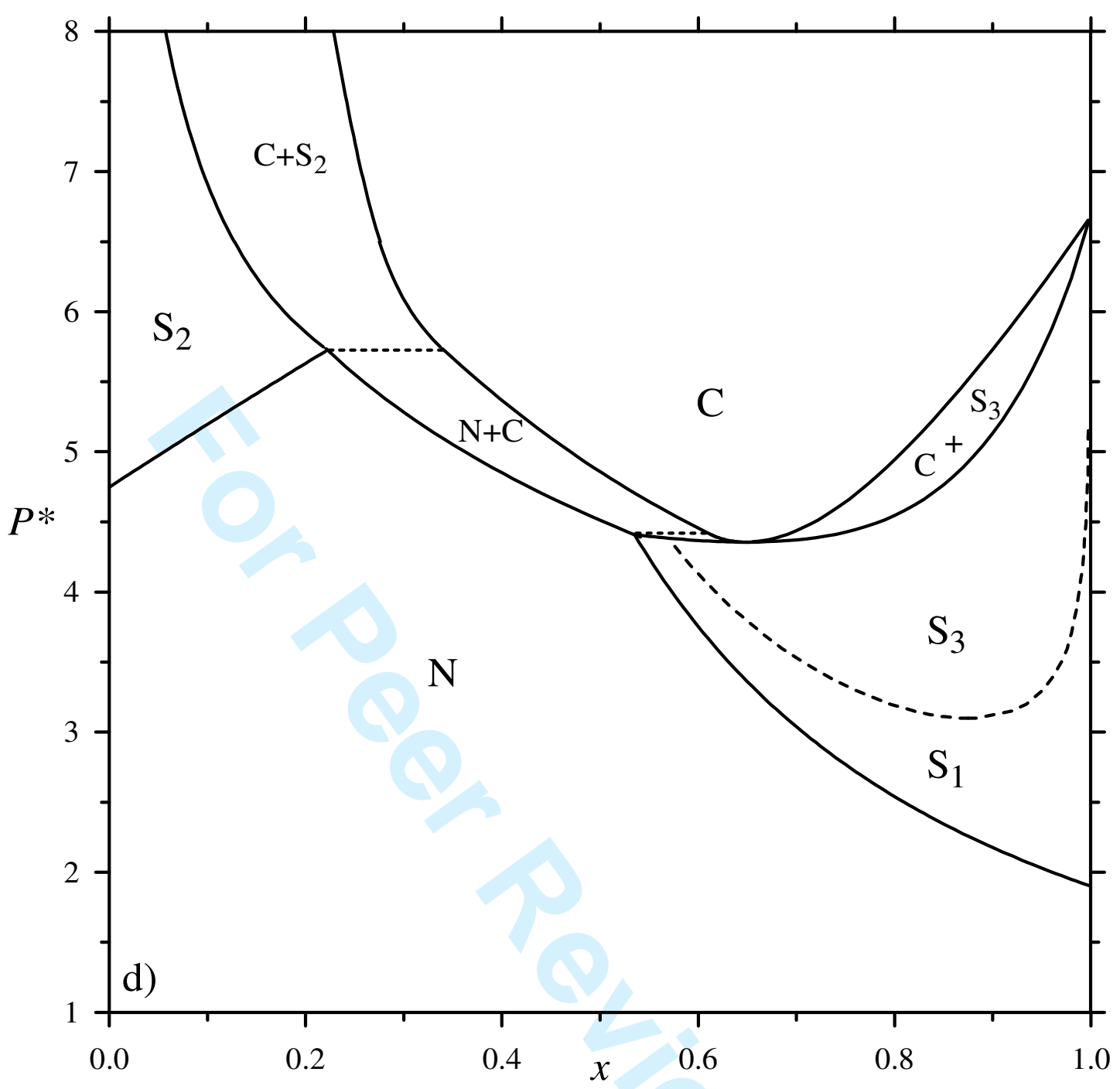

Figure 7 d) 


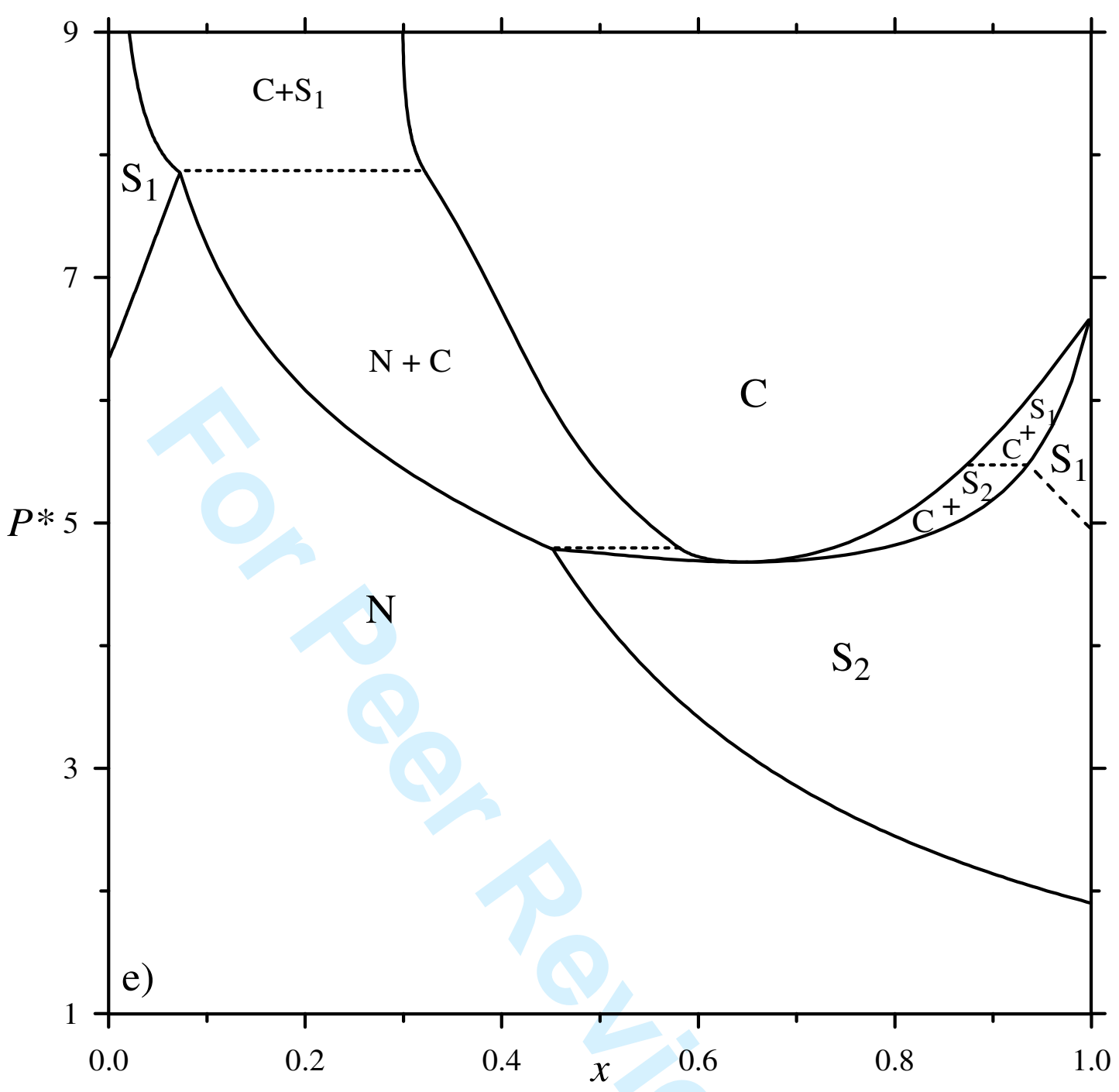

Figure 7 e) 


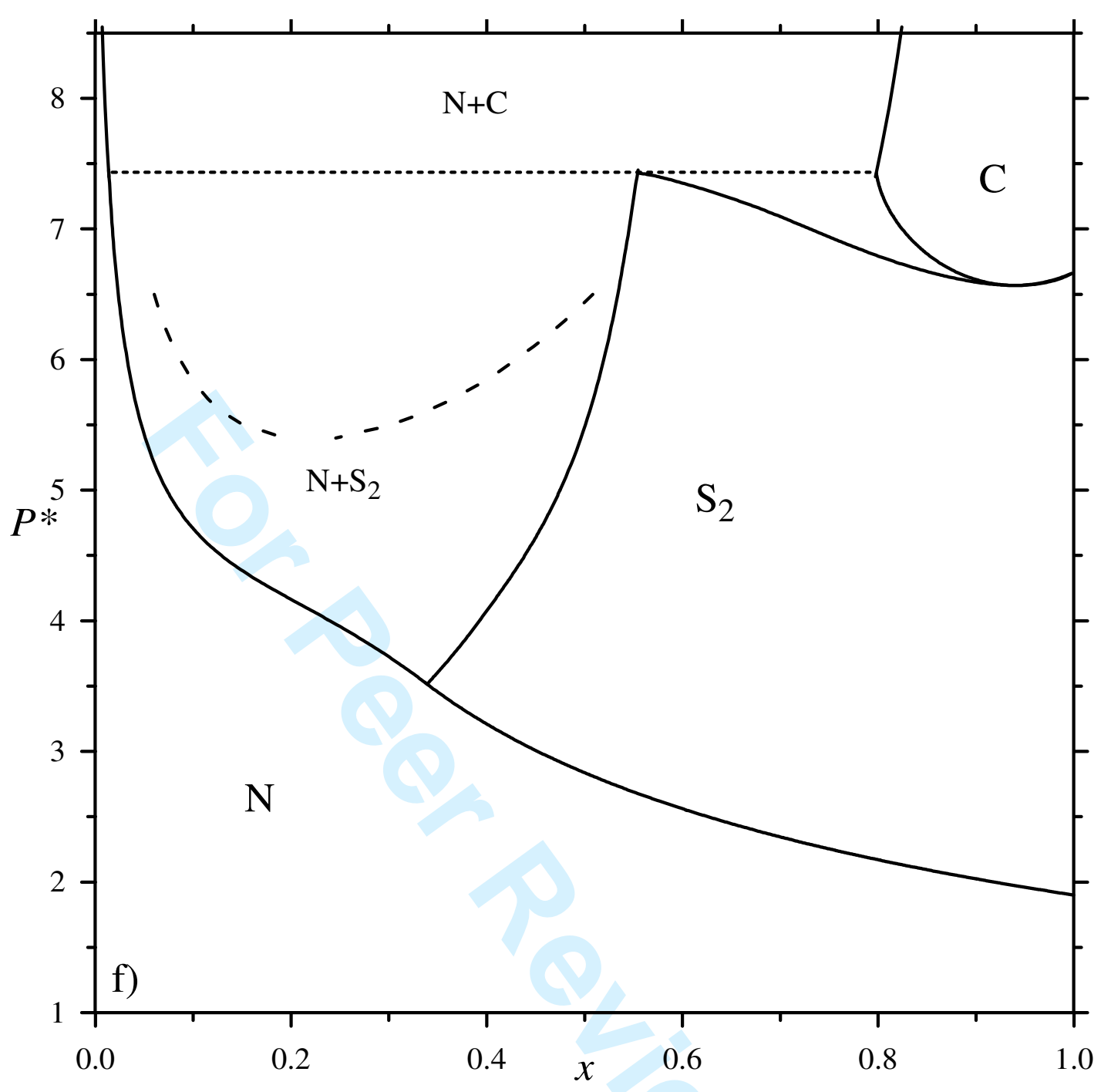

Figure 7 f) 